\title{
SMALL BALL PROBABILITIES FOR GAUSSIAN RANDOM FIELDS AND TENSOR PRODUCTS OF COMPACT OPERATORS
}

\author{
ANDREI KAROL', ALEXANDER NAZAROV, AND YAKOV NIKITIN
}

\begin{abstract}
We find the logarithmic $L_{2}$-small ball asymptotics for a large class of zero mean Gaussian fields with covariances having the structure of "tensor product". The main condition imposed on marginal covariances is the regular behavior of their eigenvalues at infinity that is valid for a multitude of Gaussian random functions including the fractional Brownian sheet, Ornstein - Uhlenbeck sheet, etc. So we get the far-reaching generalizations of well-known results by Csáki (1982) and by Li (1992). Another class of Gaussian fields considered is the class of additive fields studied under the supremum-norm by Chen and Li (2003). Our theorems are based on new results on spectral asymptotics for the tensor products of compact self-adjoint operators in Hilbert space which are of independent interest.
\end{abstract}

\section{INTRODUCTION}

The theory of small deviations of random functions is currently in intensive development. In this paper we address small deviations of Gaussian random fields in $L_{2}$-norm.

Suppose we have a Gaussian random field $X(x), x \in \Omega \subset \mathbb{R}^{d}$, with zero mean and covariance function $G_{X}(x, y)=E X(x) X(y)$ for $x, y \in \Omega$. Let $\rho$ be a summable nonnegative function on $\Omega$. Set

$$
\|X\|_{\rho}=\left(\int_{\Omega} X^{2}(x) \rho(x) d x\right)^{1 / 2}
$$

(the subscript $\rho$ will be omitted when $\rho \equiv 1$ ) and consider

$$
Q(X, \rho ; \varepsilon)=P\left\{\|X\|_{\rho} \leq \varepsilon\right\} .
$$

The problem is to define the behavior of $Q(X, \rho ; \varepsilon)$ as $\varepsilon \rightarrow 0$. Note that the case of general weight $\rho$ can be easily reduced to the case $\rho \equiv 1$ replacing $X$ by the Gaussian field $X \sqrt{\rho}$. However, it is more convenient to consider the general case.

The study of the small deviation problem was initiated by Sytaya [Sy] and continued by many scholars (see, for example, [Z1], [Z2], [DH-JS], [I], [Cs], [Li]). The history of the problem and the summary of main results can be found in two excellent reviews $[\mathrm{Lf}]$ and $[\mathrm{LiS}]$. These reviews contributed much to the considerable

Received by the editors April 24, 2005 and, in revised form, November 22, 2005.

2000 Mathematics Subject Classification. Primary 60G15; Secondary 60G60, 47A80.

Key words and phrases. Small deviations, fractional Brownian motion, Brownian sheet, Ornstein - Uhlenbeck sheet, tensor product of operators, spectral asymptotics, slowly varying functions.

The authors were partially supported by RFBR Grant 04-01-00716. 
progress lately obtained. Latest results on $L_{2}$-small deviation asymptotics for onedimensional Gaussian random functions related to Brownian motion can be found in $[\mathrm{BNO}]$, [GHT]-[GHLT2], [Na1]-[NaNi1]. Some advancements connected to fractional Brownian motion can be found in $[\mathrm{Br}]$ and [NaNi2].

Small deviations of Gaussian random functions defined on a set $\Omega \subset \mathbb{R}^{d}, d \geq 2$, are studied much less. We refer to the classical result by Csáki [Cs] who investigated the Brownian sheet $\mathbb{W}_{d}=\mathbb{W}\left(x_{1}, \ldots, x_{d}\right)$ on the unit cube. His achievements were later extended by $\mathrm{Li}[\mathrm{Li}]$ to include a larger class of random fields; see also [GL1]. The small deviation asymptotics for the Brownian sheet in the simplest case $d=2$, as given in $[\mathrm{Cs}]$, is as follows:

$$
\lim _{\varepsilon \rightarrow 0} \varepsilon^{2} \ln ^{-2}\left(1 / \varepsilon^{2}\right) \ln P\left\{\left\|\mathbb{W}_{2}\right\| \leq \varepsilon\right\}=-\frac{1}{8 \pi^{2}} .
$$

In [NaNi2] the logarithmic small deviation asymptotics was described for fractional and ordinary Lévy's random fields on arbitrary domain $\Omega \subset \mathbb{R}^{d}$.

According to the well-known Karhunen - Loève expansion which is valid also in the multiparameter case, see [Ad], we have in distribution

$$
\|X\|_{\rho}^{2}=\int_{\Omega} X^{2}(x) \rho(x) d x=\sum_{n=1}^{\infty} \lambda_{n} \xi_{n}^{2},
$$

where $\xi_{n}, n \in \mathbb{N}$, are independent standard normal r.v.'s, and $\lambda_{n}>0, n \in \mathbb{N}$, $\sum_{n} \lambda_{n}<\infty$ are the eigenvalues of the integral equation

$$
\lambda f(x)=\int_{\Omega} G_{X}(x, y) \sqrt{\rho(x) \rho(y)} f(y) d y, \quad x \in \Omega .
$$

Thus we arrive at the equivalent problem of studying the asymptotic behavior as $\varepsilon \rightarrow 0$ of $P\left\{\sum_{n=1}^{\infty} \lambda_{n} \xi_{n}^{2} \leq \varepsilon^{2}\right\}$. The answer heavily depends on the available information about the eigenvalues $\lambda_{n}$.

In this paper we study the small deviation asymptotics of a vast and important class of Gaussian random fields having the covariance of "tensor product" type. It means that this covariance can be decomposed in a product of "marginal" covariances depending on different arguments. The classical examples of such fields are the Brownian sheet, the Brownian pillow and the Brownian pillow-slip (Kiefer field), see, e.g., [vVW]. Less known examples can be found in [Kh] and [LNN]. The notion of tensor products of Gaussian processes or Gaussian measures was known long ago, see $[\mathrm{Ca}],[\mathrm{CC}]$ and $[\mathrm{Ch}]$ for a more general approach. Such Gaussian fields are also studied in related domains of mathematics, see, e.g., [PW], [RWW], [GHP], $[\mathrm{LuP} 1]$ and $[\mathrm{LuP} 2]$.

Suppose we have two Gaussian random functions $X(x), x \in \mathbb{R}^{m}$, and $Y(y)$, $y \in \mathbb{R}^{n}$, with zero means and covariances $G_{X}(x, u), x, u \in \mathbb{R}^{m}$, and $G_{Y}(y, v)$, $y, v \in \mathbb{R}^{n}$, correspondingly. Consider the new Gaussian function $Z(x, y), x \in \mathbb{R}^{m}$, $y \in \mathbb{R}^{n}$, which has zero mean and the covariance

$$
G_{Z}((x, y),(u, v))=G_{X}(x, u) G_{Y}(y, v) .
$$

Such Gaussian function obviously exists, and the integral operator with the kernel (1.5) is the tensor product of two "marginal" integral operators with the kernels $G_{X}(x, u)$ and $G_{Y}(y, v)$. Therefore we use in the sequel the notation

$$
Z=X \otimes Y
$$


and we call the Gaussian field $Z$ the tensor product of the fields $X$ and $Y$ sometimes using the shortened term field-product. The generalization to the multivariate case when obtaining the fields $\underset{j=1}{\otimes} X_{j}$ is straightforward.

In this way we can construct a large set of Gaussian random functions. The problem of asymptotic behavior for the spectrum of their covariances given we know such behavior for marginal kernels is nontrivial. One often observes for the random fields (see, e.g., $[\mathrm{PW}]$ and $[\mathrm{Li}]$ ) that the eigenvalues of marginal processes have the "pure" power asymptotics, but the asymptotics for the tensor product contain the slowly varying (logarithmic) factor.

It is natural to generalize the problem and to consider the case when the eigenvalues $\lambda_{n}^{(j)}$ of marginal covariances have the so-called regular behavior:

$$
\lambda_{n}^{(j)} \sim \frac{\varphi_{j}(n)}{n^{p_{j}}}, n \rightarrow \infty
$$

where $p_{j}>1$ are called the marginal exponents and $\varphi_{j}$ are some slowly varying functions (SVFs), in particular, the powers of logarithmic function. The assumption of regular behavior is common in recent papers using spectral asymptotics, see, e.g., [BS3], [De], [LuP1] and [LuP2].

Gaussian processes with regular spectral asymptotics can be easily constructed. Let $X(x), x \in[0,1]$, be the stationary Gaussian process with zero mean and covariance

$$
G_{X}(x, y)=g(|x-y|),
$$

where $g$ is a smooth function on $\mathbb{R}_{+}$, and

$$
g(x)-g(0)=-x^{\alpha} \varphi(1 / x)+O\left(x^{\beta}\right), \quad x \rightarrow 0+,
$$

where $0<\alpha<2, \beta>\alpha$, while $\varphi$ is a SVF. Then, similarly to [BS1], Appendix 7, one can show that

$$
\lambda_{n} \sim \frac{2 \sin \frac{\pi \alpha}{2} \Gamma(\alpha+1)}{\pi^{\alpha+1}} \cdot \frac{\varphi(n)}{n^{1+\alpha}}, \quad n \rightarrow \infty .
$$

We also note that some simple Gaussian processes with nonregular spectral asymptotics were considered in [Na2].

The problem of small ball behavior for fields-products when the eigenvalues of their factors are decreasing as in (1.6) is considerably more difficult than in the case of purely power decrease. To solve it, we develop a new method. It is based on abstract theorems describing the spectral asymptotics of tensor products and of sums of tensor products for self-adjoint operators in Hilbert space proved below. They contain as a special case the results from [PW], see also [LuP2], Proposition 3.4, where the factors of the tensor product have purely power decrease of eigenvalues.

Two essentially different cases arise in our theorems on spectral asymptotics of tensor products. The first case deals with the equal marginal exponents $p_{i} \equiv p$ of the factors in the field-product like in the case of a Brownian sheet or a fractional Brownian sheet with same Hurst parameters.

The second one deals with the different marginal exponents of these factors. The situation there is more delicate. The order of logarithmic small ball asymptotics is defined by the "factor" with the slowest marginal exponent. However, in order to find the logarithmic small ball constants we usually need the exact form of the 
spectrum for the "factors" with fast decrease. Similar effects were discovered in the particular case in [Li], Example 2.

The examples of Gaussian processes with exactly known spectrum are rather numerous. We mention here the Brownian motion, the Brownian bridge, the centered Brownian motion, the centered Brownian bridge and some other processes, see $\S 6$. If one such process serves as the fast factor, then the logarithmic small ball constant of the slow factor should be multiplied by the value of Riemann zetafunction in a particular point.

It is worth mentioning that the parallel development of small deviation asymptotics for Gaussian fields in the supremum norm also came across the substantial difference between fractional Brownian sheets with equal and distinct factors, see, e.g., [MSh], [BL], [KLn], [GL1] and [GL2].

Recently Fill and Torcaso [FT] obtained the exact asymptotics of small deviations in $L_{2}$-norm for the Brownian sheet and its integrated counterparts. Their original approach is based on the Mellin transform and is entirely different from our method. It is not clear if their approach makes it possible to obtain small deviation results for a vast class of Gaussian fields-products studied in the present paper when the eigenvalues satisfy (1.6). Moreover, our results are applicable for the weighted $L_{2}$ norm in the case of general (continuous) weights on the unit cube in $\mathbb{R}^{d}$. This seems to be beyond the reach of the method using Mellin transform.

Another type of Gaussian fields we consider is the class of additive processes, see [CL]. For short, we often call it fields-sums. Let $X_{j}\left(x_{j}\right), 1 \leq j \leq d, x_{j} \in[0,1]^{\ell_{j}}$, be independent random functions. The additive process $\mathbb{X}(x)$ is defined as

$$
\mathbb{X}(x)=\mathbb{X}\left(x_{1}, \ldots, x_{d}\right)=\sum_{j=1}^{d} X_{j}\left(x_{j}\right), \quad x=\left(x_{1}, \ldots, x_{d}\right) \in[0,1]^{D},
$$

where $D=\sum_{j=1}^{d} \ell_{j}$.

There are various motivations for the study of additive processes: they are relatively simple approximations to more complicated multiparameter processes and appear, e.g., in the theory of intersections and selfintersections of Brownian processes. Some small deviation results with respect to supremum norm were proved in [CL]. The results in the $L_{2}$-norm obtained below appear for the first time.

The structure of the paper is as follows. First, in $\S 2$ we present some auxiliary information about SVFs. The results are partly borrowed from [Se] but some of them seem to be new such as the useful properties of Mellin convolution of SVFs.

Next, in $\S 3$ we prove new results on the spectral asymptotics for tensor products of compact self-adjoint operators in Hilbert spaces and for sums of such tensor products. These results are adapted for the applications to small ball asymptotics but are of independent interest.

In $\S 4$ we derive the logarithmic small ball asymptotics for Gaussian random functions with regularly varying eigenvalues. In particular, we evaluate the small ball constants for the special rate of eigenvalues decrease, namely

$$
\lambda_{n} \sim C^{*} \frac{\ln ^{\sigma}(n+1)}{n^{p}}, \quad \sigma \in \mathbb{R}, \quad p>1, \quad n \rightarrow \infty .
$$

The case $\sigma=0$ is well known while in the presence of logarithmic factor the results are new. We also obtain a weaker result (namely, the double logarithmic asymptotics) for the special case $p=1$. 
We then apply the elaborated theory to various concrete examples of Gaussian random fields. We treat in $\S 5$ the fields-products with equal marginal exponents and in $\S 6$ the fields-products with distinct marginal exponents. The fields-sums are studied in $\S 7$.

In $\S 8$ we substantially enlarge the class of weights $\rho$ in (1.1) for which the results of $\S 5$ are still valid. We prove that instead of the products of univariate weights one can take any continuous weight on the unit cube in $\mathbb{R}^{d}$. Finally, in the same section we consider various generalizations to the vector-valued random fields.

A preliminary version of this paper with considerably less general statements was published as a preprint $[\mathrm{KNN}]$. Similar results using a more probabilistic approach were obtained in [GL2].

\section{Auxiliary information on SVFs}

We recall that a positive function $\varphi(\tau), \tau>0$, is called a slowly varying function $(\mathrm{SVF})$ at infinity if for any $c>0$

$$
\varphi(c \tau) / \varphi(\tau) \rightarrow 1 \quad \text { as } \quad \tau \rightarrow+\infty .
$$

Remark 1. Clearly, any smooth positive function $\varphi$ satisfying $\tau \varphi^{\prime}(\tau) / \varphi(\tau) \rightarrow 0$ as $\tau \rightarrow+\infty$ is slowly varying. This test shows that the functions equal to $\ln ^{\alpha}(\tau)$, $\ln ^{\alpha}(\tau) \ln ^{\alpha_{1}}(\ln (\tau))$, etc. for $\tau \gg 1$ are slowly varying. However, one must keep in mind that SVFs can be less regular (see [BS3]): the SVF $\varphi(\tau)=2+\sin \left(\ln ^{\alpha}(\tau)\right.$ ), $\alpha<1$, is bounded and separated from zero, but it has no limit as $\tau \rightarrow+\infty$; the SVF $\varphi(\tau)=1+\ln (\ln (\tau)) \sin ^{2}(\ln (\ln (\ln (\tau))))$ is unbounded but does not tend to infinity as $\tau \rightarrow+\infty$.

We need some simple properties of SVFs. Their proofs can be found, for example, in $[\mathrm{Se}]$.

Proposition 2.1. Let $\varphi$ be a SVF. Then the following statements are true:

1. The relation (2.1) is uniform with respect to $c \in[a, b]$ for $0<a<b<+\infty$.

2. There exists $\tau_{0}$ such that

$$
\varphi(\tau)=\eta(\tau) \cdot \exp \left(\int_{\tau_{0}}^{\tau} \frac{\theta(\sigma)}{\sigma} d \sigma\right), \quad \tau>\tau_{0} .
$$

The function $\eta(\tau)$ has a nonzero limit as $\tau \rightarrow+\infty$ while $\theta(\sigma) \rightarrow 0$ as $\sigma \rightarrow+\infty$. Later we assume, without loss of generality, that $\tau_{0}=1$.

3. The function $\tau \mapsto \tau^{p} \varphi(\tau), p \neq 0$, is monotone for large $\tau$, and its inverse function is

$$
\tau \mapsto \tau^{1 / p} \phi(\tau), \quad p>0 ; \quad \tau \mapsto \tau^{1 / p} \phi(1 / \tau), \quad p<0,
$$

where $\phi$ is a SVF.

4. There exists an equivalent $S V F \psi \in \mathcal{C}^{2}\left(\mathbb{R}_{+}\right)($i.e. $\psi(\tau) / \varphi(\tau) \rightarrow 1$ as $\tau \rightarrow+\infty$ ) such that

$$
\tau \cdot(\ln (\psi))^{\prime}(\tau) \rightarrow 0, \quad \tau^{2} \cdot(\ln (\psi))^{\prime \prime}(\tau) \rightarrow 0, \quad \tau \rightarrow+\infty .
$$


5. If

$$
\int_{1}^{\infty} \varphi(\tau) \frac{d \tau}{\tau}<\infty
$$

then $\varphi(\tau) \rightarrow 0$ as $\tau \rightarrow+\infty$

We define the Mellin convolution of two $\operatorname{SVFs} \varphi$ and $\psi$ as

$$
(\varphi * \psi)(\tau)=\int_{1}^{\tau} \varphi(\sigma) \psi(\tau / \sigma) \frac{d \sigma}{\sigma}=h_{\varphi, \psi}(\tau)+h_{\psi, \varphi}(\tau),
$$

where

$$
h_{\varphi, \psi}(\tau)=\int_{1}^{\sqrt{\tau}} \varphi(\sigma) \psi(\tau / \sigma) \frac{d \sigma}{\sigma} .
$$

Theorem 2.2. The following statements are true:

1. If (2.4) holds, and

$$
\int_{1}^{\infty} \varphi(\sigma) m_{\psi}(\sigma) \frac{d \sigma}{\sigma}<\infty
$$

where

$$
m_{\psi}(\sigma)=\sup _{\tau>\sigma^{2}} \frac{\psi(\tau / \sigma)}{\psi(\tau)}
$$

then

$$
h_{\varphi, \psi}(\tau)=\psi(\tau) \int_{1}^{\infty} \varphi(\sigma) \frac{d \sigma}{\sigma} \cdot(1+o(1)), \quad \tau \rightarrow+\infty .
$$

2. If $\int_{1}^{\infty} \varphi(\sigma) \frac{d \sigma}{\sigma}=\infty$, then $\psi(\tau)=o\left(h_{\varphi, \psi}(\tau)\right)$ as $\tau \rightarrow+\infty$.

3. If $\psi(\tau)=\psi_{1}(\tau) \cdot(1+o(1)), \tau \rightarrow+\infty$, then

$$
h_{\varphi, \psi}(\tau)=h_{\varphi, \psi_{1}}(\tau) \cdot(1+o(1)), \quad \tau \rightarrow+\infty .
$$

If, in addition, $\int_{1}^{\infty} \psi(\sigma) \frac{d \sigma}{\sigma}=\infty$, then

$$
h_{\psi, \varphi}(\tau)=h_{\psi_{1}, \varphi}(\tau) \cdot(1+o(1)), \quad \tau \rightarrow+\infty .
$$

4. $h_{\varphi, \psi}$ is a $S V F$.

Proof. 1. We have

$$
h_{\varphi, \psi}(\tau)=\psi(\tau) \int_{1}^{\sqrt{\tau}} \varphi(\sigma) \frac{\psi(\tau / \sigma)}{\psi(\tau)} \frac{d \sigma}{\sigma} .
$$

By Lebesgue Dominated Convergence Theorem, the statement follows.

2. This statement follows from elementary inequality

$$
h_{\varphi, \psi}(\tau)>\int_{1}^{a} \varphi(\sigma) \psi(\tau / \sigma) \frac{d \sigma}{\sigma}=\psi(\tau) \int_{1}^{a} \varphi(\sigma) \frac{d \sigma}{\sigma} \cdot(1+o(1)), \quad \tau \rightarrow+\infty .
$$


3. The first property is quite elementary. To prove the second one we note that due to $\mathbf{2}$ for any $a>1$

$$
h_{\psi, \varphi}(\tau)=\int_{a}^{\sqrt{\tau}} \psi(\sigma) \varphi(\tau / \sigma) \frac{d \sigma}{\sigma} \cdot(1+o(1)), \quad \tau \rightarrow \infty .
$$

Given $\varepsilon>0$, one can take $a$ so large that $1-\varepsilon<\psi(\sigma) / \psi_{1}(\sigma)<1+\varepsilon$ for $\sigma>a$, and the statement follows.

4. Due to 3 and to Proposition 2.1, part 4, we can assume $\psi$ smooth. We have

$$
\tau h_{\varphi, \psi}^{\prime}(\tau)=\frac{1}{2} \varphi(\sqrt{\tau}) \psi(\sqrt{\tau})+\int_{1}^{\sqrt{\tau}} \varphi(\sigma) \psi^{\prime}(\tau / \sigma) \frac{\tau}{\sigma} \cdot \frac{d \sigma}{\sigma} .
$$

For any $a>1$ and $\tau>a^{2}$

$$
h_{\varphi, \psi}(\tau)>\int_{a^{-1} \sqrt{\tau}}^{\sqrt{\tau}} \varphi(\sigma) \psi(\tau / \sigma) \frac{d \sigma}{\sigma}=\int_{a^{-1}}^{1} \varphi(\sqrt{\tau} \sigma) \psi(\sqrt{\tau} / \sigma) \frac{d \sigma}{\sigma} .
$$

By Proposition 2.1, part 1, the integrand converges uniformly as $\tau \rightarrow+\infty$, and we obtain

$$
h_{\varphi, \psi}(\tau)>\varphi(\sqrt{\tau}) \psi(\sqrt{\tau}) \ln (a) \cdot(1+o(1))
$$

Hence

$$
\varphi(\sqrt{\tau}) \psi(\sqrt{\tau})=o\left(h_{\varphi, \psi}(\tau)\right), \quad \tau \rightarrow+\infty .
$$

Next, due to (2.3) we have

$$
\left|\int_{1}^{\sqrt{\tau}} \varphi(\sigma) \psi^{\prime}(\tau / \sigma) \frac{\tau}{\sigma} \cdot \frac{d \sigma}{\sigma}\right| \leq \sup _{\lambda \geq \sqrt{\tau}}\left|\frac{\lambda \psi^{\prime}(\lambda)}{\psi(\lambda)}\right| \cdot h_{\varphi, \psi}(\tau)=o\left(h_{\varphi, \psi}(\tau)\right), \quad \tau \rightarrow+\infty .
$$

So, $\tau h_{\varphi, \psi}^{\prime}(\tau)=o\left(h_{\varphi, \psi}(\tau)\right)$, and $\mathbf{4}$ follows.

Remark 2. We underline that the condition (2.4) does not imply $\left(2.5_{\varphi \psi}\right)$ without additional assumptions. Also in the general case $h_{\psi, \varphi}(\tau) \neq h_{\psi_{1}, \varphi}(\tau) \cdot(1+o(1))$ as $\tau \rightarrow+\infty$.

Example 1. Let $\varphi(\tau)=(1+\ln (\tau))^{\varkappa_{1}}, \psi(\tau)=(1+\ln (\tau))^{\varkappa_{2}}$. One can check by direct calculation that, as $\tau \rightarrow+\infty$,

$$
(\varphi * \psi)(\tau) \sim \begin{cases}\mathbf{B}\left(\varkappa_{1}+1, \varkappa_{2}+1\right)(1+\ln (\tau))^{\varkappa_{1}+\varkappa_{2}+1}, & \varkappa_{1}>-1, \varkappa_{2}>-1 ; \\ \ln (\ln (\tau)) \cdot(1+\ln (\tau))^{\varkappa_{2}}, & \varkappa_{1}=-1, \varkappa_{2}>-1 ; \\ \frac{1}{\mid \varkappa_{1}+1} \cdot(1+\ln (\tau))^{\varkappa_{2}}, & \varkappa_{1}<-1, \varkappa_{1}<\varkappa_{2} \\ 2 \ln (\ln (\tau)) \cdot(1+\ln (\tau))^{-1}, & \varkappa_{1}=\varkappa_{2}=-1 \\ \frac{2}{\left|\varkappa_{1}+1\right|} \cdot(1+\ln (\tau))^{\varkappa_{1}}, & \varkappa_{1}=\varkappa_{2}<-1\end{cases}
$$

where $\mathbf{B}$ is the Euler beta-function. Note that in the last two cases the factor 2 arises because the functions $h_{\varphi, \psi}(\tau)$ and $h_{\psi, \varphi}(\tau)$ have the same asymptotics. 


\section{Spectral ASYMptotics FOR tensor Products OF COMPACT SELF-ADJOINT OPERATORS}

Let $\mathcal{T}=\mathcal{T}^{*} \geq 0$ be a compact self-adjoint nonnegative operator in a Hilbert space $H$; let $\lambda_{n}=\lambda_{n}(\mathcal{T})$ be its positive eigenvalues arranged in a nondecreasing sequence and repeated according to their multiplicity.

In spectral theory it is common and convenient considering the counting function

$$
\mathcal{N}(t)=\mathcal{N}(t, \mathcal{T})=\#\left\{n: \lambda_{n}(\mathcal{T})>t\right\} .
$$

We formulate some of its properties which are necessary for the subsequent arguments. Their proof is quite standard, and we omit it.

Lemma 3.1. The following statements are true:

1. The relation $\mathcal{N}(t, \mathcal{T})=O\left(t^{-1 / p}\right), t \rightarrow 0+$, is equivalent to $\lambda_{n}(\mathcal{T})=O\left(n^{-p}\right)$, $n \rightarrow \infty$.

2. The asymptotics

$$
\mathcal{N}(t, \mathcal{T}) \sim \mathcal{N}_{a s}(t) \equiv a \cdot \frac{\varphi(1 / t)}{t^{1 / p}}, \quad t \rightarrow 0+,
$$

(here $p>0$ while $\varphi$ is a SVF) is equivalent to the spectral asymptotics

$$
\lambda_{n}(\mathcal{T}) \sim \frac{\psi(n)}{n^{p}}, \quad n \rightarrow \infty
$$

where $\psi$ is also a SVF.

3. Let $\mathcal{T}$ have the counting function with the asymptotics (3.1). Then the convergence of the integral $\int_{1}^{\infty} \varphi(\tau) \frac{d \tau}{\tau}$ is equivalent to the convergence of the series $\sum_{n} \lambda_{n}^{1 / p}(\mathcal{T})$.

Example 2. The asymptotics

$$
\mathcal{N}(t, \mathcal{T}) \sim a \frac{\ln ^{\varkappa}(1 / t)}{t^{1 / p}}, \quad t \rightarrow 0+,
$$

with $p>0$, is equivalent to the spectral asymptotics

$$
\lambda_{n}(\mathcal{T}) \sim a^{p} p^{p \varkappa} \frac{\ln ^{p \varkappa}(n+1)}{n^{p}}, \quad n \rightarrow \infty .
$$

Note that in view of Proposition 2.1, part 4, any SVF arising in an asymptotic formula can be assumed smooth. Moreover, we can take $\eta \equiv 1$ in the representation formula $(2.2)$.

We introduce the functionals

$$
\Delta_{p, \varphi}(\mathcal{T})=\limsup _{t \rightarrow 0+} \frac{t^{1 / p}}{\varphi(1 / t)} \cdot \mathcal{N}(t, \mathcal{T}), \quad \delta_{p, \varphi}(\mathcal{T})=\liminf _{t \rightarrow 0+} \frac{t^{1 / p}}{\varphi(1 / t)} \cdot \mathcal{N}(t, \mathcal{T}) .
$$

The asymptotics $(3.1)$ means that $\Delta_{p, \varphi}(\mathcal{T})=\delta_{p, \varphi}(\mathcal{T})$ are finite and are not equal to zero.

Note that due to (2.1) for any positive $c$ we have

$$
\Delta_{p, \varphi}(c \mathcal{T})=c^{1 / p} \Delta_{p, \varphi}(\mathcal{T}), \quad \delta_{p, \varphi}(c \mathcal{T})=c^{1 / p} \delta_{p, \varphi}(\mathcal{T}) .
$$

Theorem 3.2. Let operator $\mathcal{T}$ have the counting function with asymptotics (3.1). Let $\widetilde{\mathcal{T}}$ be a compact self-adjoint nonnegative operator in a Hilbert space $\widetilde{H}$ with

$$
\widetilde{\mathcal{N}}(t) \equiv \mathcal{N}(t, \widetilde{\mathcal{T}})=O\left(t^{-1 / \widetilde{p}}\right), \quad t \rightarrow 0+, \quad \widetilde{p}>p .
$$


Then the operator $\mathcal{T} \otimes \widetilde{\mathcal{T}}$ in the space $H \otimes \widetilde{H}$ satisfies the relation

$$
\mathcal{N}_{\otimes}(t) \equiv \mathcal{N}(t, \mathcal{T} \otimes \widetilde{\mathcal{T}}) \sim a \cdot \frac{\varphi(1 / t)}{t^{1 / p}} \sum_{n} \lambda_{n}^{1 / p}(\widetilde{\mathcal{T}}), \quad t \rightarrow 0+.
$$

Proof. As the eigenvalues of $\mathcal{T} \otimes \widetilde{\mathcal{T}}$ are the products of $\lambda_{j}=\lambda_{j}(\mathcal{T})$ and $\widetilde{\lambda}_{k}=\lambda_{k}(\tilde{\mathcal{T}})$ we have

$$
\mathcal{N}_{\otimes}(t)=\#\left\{k, j: \quad \lambda_{j} \tilde{\lambda}_{k}>t\right\}=\sum_{k} \#\left\{j: \lambda_{j}>t / \tilde{\lambda}_{k}\right\}=\sum_{k} \mathcal{N}\left(t / \tilde{\lambda}_{k}\right) .
$$

Thus, we obtain

$$
\frac{t^{1 / p}}{\varphi(1 / t)} \cdot \mathcal{N}_{\otimes}(t)=\sum_{k}\left[\frac{\left(t / \widetilde{\lambda}_{k}\right)^{1 / p}}{\varphi\left(\widetilde{\lambda}_{k} / t\right)} \cdot \mathcal{N}\left(t / \widetilde{\lambda}_{k}\right)\right] \cdot \frac{\varphi\left(\widetilde{\lambda}_{k} / t\right)}{\varphi(1 / t)} \cdot \widetilde{\lambda}_{k}^{1 / p}
$$

Due to (3.1) the term in square brackets is uniformly bounded and tends to $a$ as $t \rightarrow 0+$. The second quotient in the sum tends to 1 as $\varphi$ is a SVF.

Next, by Proposition 2.1, part 3, for any $\varepsilon>0 \tau^{\varepsilon} \varphi(\tau)$ increases for $\tau>\tau_{0}(\varepsilon)$. Hence we have the inequality

$$
\frac{\lambda^{\varepsilon} \varphi(\lambda \tau)}{\varphi(\tau)}=\frac{(\lambda \tau)^{\varepsilon} \varphi(\lambda \tau)}{\tau^{\varepsilon} \varphi(\tau)} \leq 1
$$

for $\lambda \tau>\tau_{0}(\varepsilon), \lambda \leq 1$. So for any $\varepsilon>0$ the estimate

$$
\frac{\varphi\left(\widetilde{\lambda}_{k} / t\right)}{\varphi(1 / t)} \leq C(\varepsilon) \widetilde{\lambda}_{k}^{-\varepsilon}
$$

holds uniformly for $t<1$. Using the estimate (3.3) we get

$$
\frac{\varphi\left(\widetilde{\lambda}_{k} / t\right)}{\varphi(1 / t)} \cdot \widetilde{\lambda}_{k}^{1 / p} \leq C_{1}(\varepsilon) k^{-\widetilde{p}\left(p^{-1}-\varepsilon\right)} .
$$

For sufficiently small $\varepsilon$ we observe that $\widetilde{p}\left(p^{-1}-\varepsilon\right)>1$. This gives the majorant required in the Lebesgue Theorem. Passing to the limit in the sum we get the asymptotics (3.4).

Remark 3. If $\widetilde{\mathcal{T}}$ is a finite-dimensional operator, then the estimate (3.3) holds true for any $\widetilde{p}>0$ and the sum in (3.4) is finite.

Theorem 3.3. Let the operator $\mathcal{T}$ be as in Theorem 3.2. Let the counting function of the operator $\widetilde{\mathcal{T}}$ in the space $\widetilde{H}$ have the analogous asymptotics:

$$
\tilde{\mathcal{N}}(t) \sim \widetilde{\mathcal{N}}_{a s}(t) \equiv \widetilde{a} \cdot \frac{\widetilde{\varphi}(1 / t)}{t^{1 / p}}, \quad t \rightarrow 0+.
$$

Then for any $\varepsilon>0$ the distribution function $\mathcal{N}_{\otimes}(t)$ satisfies the following estimates:

$$
\mathcal{N}_{\otimes}(t) \lessgtr \frac{\alpha_{ \pm}(\varepsilon)}{t^{1 / p}} \cdot\left[S(t, \varepsilon)+\widetilde{S}(t, \varepsilon)+\frac{a \widetilde{a}}{p} \int_{\alpha_{\mp}(\varepsilon) / \varepsilon}^{\varepsilon \tau} \varphi\left(\frac{\tau}{\sigma}\right) \widetilde{\varphi}(\sigma) \frac{d \sigma}{\sigma}\right]
$$

uniformly with respect to $t>0$ (here $\tau$ stands for $\left.\alpha_{ \pm}(\varepsilon) t^{-1}\right)$. For $\alpha_{\mp}(\varepsilon) / \varepsilon>\varepsilon \tau$ the integral in (3.6) must be omitted. 
In (3.6) $\alpha_{ \pm}(\varepsilon) \rightarrow 1$ as $\varepsilon \rightarrow 0+$, and the functions $S, \widetilde{S}$ have the following asymptotics as $t \rightarrow 0+$ :

$$
\begin{gathered}
S(t, \varepsilon) \sim a \varphi(1 / t) \cdot \sum_{\widetilde{\lambda}_{k} \geq \varepsilon} \widetilde{\lambda}_{k}^{1 / p}, \\
\widetilde{S}(t, \varepsilon) \sim \widetilde{a} \widetilde{\varphi}(1 / t) \cdot\left(\sum_{\lambda_{k} \geq \varepsilon} \lambda_{k}^{1 / p}+a \varphi(1 / \varepsilon)\right) .
\end{gathered}
$$

Proof. We establish the upper bound for $\mathcal{N}_{\otimes}(t)$. The lower estimate can be proved in the same way. We have

$$
t^{1 / p} \mathcal{N}_{\otimes}(t)=t^{1 / p} \sum_{k} \mathcal{N}\left(t / \widetilde{\lambda}_{k}\right)=t^{1 / p} \sum_{\widetilde{\lambda}_{k}<\varepsilon} \mathcal{N}\left(t / \widetilde{\lambda}_{k}\right)+S(t, \varepsilon),
$$

where

$$
S(t, \varepsilon)=t^{1 / p} \cdot \sum_{\tilde{\lambda}_{k} \geq \varepsilon} \mathcal{N}\left(t / \widetilde{\lambda}_{k}\right) .
$$

The asymptotics (3.7a) for the last sum follows from Theorem 3.2.

Denote by $\widetilde{\mu}$ the inverse function for $\widetilde{\mathcal{N}}_{\text {as }}$. Then (3.5) implies $\widetilde{\lambda}_{k} / \widetilde{\mu}(k) \rightarrow 1$ as $k \rightarrow \infty$, and we have

$$
\alpha_{-}(\varepsilon) \widetilde{\mu}(k) \leq \widetilde{\lambda}_{k} \leq \alpha_{+}(\varepsilon) \widetilde{\mu}(k)
$$

for $\widetilde{\lambda}_{k}<\varepsilon$, with $\alpha_{ \pm}(\varepsilon) \rightarrow 1$ as $\varepsilon \rightarrow 0$.

Using monotonicity of $\mathcal{N}$ we get

$$
\sum_{\widetilde{\lambda}_{k}<\varepsilon} \mathcal{N}\left(t / \widetilde{\lambda}_{k}\right) \leq \sum_{\widetilde{\mu}(k)<\varepsilon \alpha_{-}^{-1}(\varepsilon)} \mathcal{N}\left(t / \alpha_{+}(\varepsilon) \widetilde{\mu}(k)\right),
$$

and by monotonicity of the function $k \mapsto \mathcal{N}\left(t / \alpha_{+}(\varepsilon) \widetilde{\mu}(k)\right)$

$$
t^{1 / p} \sum_{\widetilde{\lambda}_{k}<\varepsilon} \mathcal{N}\left(t / \widetilde{\lambda}_{k}\right) \leq t^{1 / p} \mathcal{N}\left(\frac{\alpha_{-}(\varepsilon) t}{\alpha_{+}(\varepsilon) \varepsilon}\right)+t^{1 / p} \int_{0}^{\varepsilon \alpha_{-}^{-1}(\varepsilon)} \mathcal{N}\left(t / \alpha_{+}(\varepsilon) \mu\right)\left(-d \mathcal{N}_{a s}(\mu)\right)
$$

The first term in (3.8) is $O\left(\varepsilon^{1 / p} \varphi(1 / t)\right)$. Therefore, adding it to the term $S(t, \varepsilon)$, we obtain the term $\alpha_{+}(\varepsilon) S(t, \varepsilon)$ with $\alpha_{+}(\varepsilon) \rightarrow 1$ as $\varepsilon \rightarrow 0$. Further,

$$
-d \mathcal{N}_{a s}(\mu)=\widetilde{a} \cdot \frac{\widetilde{\varphi}(1 / \mu)}{\mu^{1+1 / p}} \cdot\left(\frac{1}{p}+\widetilde{\theta}(1 / \mu)\right) d \mu,
$$

where $\tilde{\theta}$ is the function from the formula $(2.2)$ for $\operatorname{SVF} \widetilde{\varphi}$. Since $\widetilde{\theta}(1 / \mu) \rightarrow 0$ as $\mu \rightarrow 0$, we can estimate the integral term in (3.8) by

$$
\alpha_{+}(\varepsilon) \frac{\widetilde{a}}{p} t^{1 / p} \int_{0}^{\varepsilon \alpha_{-}^{-1}(\varepsilon)} \mathcal{N}\left(t / \alpha_{+}(\varepsilon) \mu\right) \frac{\widetilde{\varphi}(1 / \mu)}{\mu^{1+1 / p}} d \mu .
$$

Splitting the integral in (3.9) and changing variables we obtain

$$
\alpha_{+}(\varepsilon) \frac{\widetilde{a}}{p} \cdot \int_{\varepsilon}^{\infty} \mathcal{N}(s) \frac{\widetilde{\varphi}\left(\alpha_{+}(\varepsilon) s / t\right)}{s^{1-1 / p}} d s+\alpha_{+}(\varepsilon) \frac{\widetilde{a}}{p} t^{1 / p} \int_{\alpha_{-}(\varepsilon) / \varepsilon}^{\varepsilon \tau} \mathcal{N}(\sigma / \tau) \frac{\widetilde{\varphi}(\sigma)}{\sigma^{1-1 / p}} d \sigma .
$$


The first integral in (3.10) gives us the term $\widetilde{S}(t, \varepsilon)$. By Proposition 2.1, part 1, $\frac{\widetilde{\varphi}\left(\alpha_{+}(\varepsilon) s / t\right)}{\widetilde{\varphi}(1 / t)} \rightarrow 1$ as $t \rightarrow 0$ uniformly with respect to $s \in[\varepsilon,\|T\|]$ (for $s>\|T\|$ the integrand equals 0 ), and we have

$$
\widetilde{S}(t, \varepsilon) \sim \widetilde{a} \widetilde{\varphi}(1 / t) \cdot \int_{\varepsilon}^{\infty} \mathcal{N}(s) d s^{1 / p} .
$$

Integrating by parts we arrive at (3.7b).

Due to (3.1) we can estimate $\mathcal{N}$ in the second integral by $\alpha_{+}(\varepsilon) \mathcal{N}_{a s}$ that gives the integral term in (3.6).

Theorem 3.4. Let operators $\mathcal{T}$ and $\widetilde{\mathcal{T}}$ be as in Theorem 3.3.

1. If

$$
\int_{1}^{\infty} \varphi(\tau) \frac{d \tau}{\tau}=\int_{1}^{\infty} \tilde{\varphi}(\tau) \frac{d \tau}{\tau}=\infty
$$

then

$$
\mathcal{N}_{\otimes}(t) \sim \frac{\phi(1 / t)}{t^{1 / p}}, \quad t \rightarrow 0
$$

where

$$
\phi(s)=\frac{a \widetilde{a}}{p}(\varphi * \widetilde{\varphi})(s) .
$$

2. If

$$
\int_{1}^{\infty} \varphi(\tau) \frac{d \tau}{\tau}<\infty, \quad \int_{1}^{\infty} \widetilde{\varphi}(\tau) \frac{d \tau}{\tau}=\infty,
$$

and the asymptotic formula (2.5 $\widetilde{\varphi} \varphi$ ) is fulfilled, then (3.12) holds true with

$$
\phi(s)=\widetilde{a} \widetilde{\varphi}(s) \sum_{n} \lambda_{n}^{1 / p}+\frac{a \widetilde{a}}{p} h_{\widetilde{\varphi}, \varphi}(s) .
$$

3. If

$$
\int_{1}^{\infty} \varphi(\tau) \frac{d \tau}{\tau}<\infty, \quad \int_{1}^{\infty} \widetilde{\varphi}(\tau) \frac{d \tau}{\tau}<\infty,
$$

and the asymptotic formulas (2.5 $\left.\tilde{\varphi}_{\tilde{\varphi}}\right),\left(2.5_{\widetilde{\varphi} \varphi}\right)$ are fulfilled, then (3.12) holds true with

$$
\phi(s)=a \varphi(s) \sum_{n} \widetilde{\lambda}_{n}^{1 / p}+\widetilde{a} \widetilde{\varphi}(s) \sum_{n} \lambda_{n}^{1 / p} .
$$

Proof. 1. Fix arbitrary $\varepsilon>0$ and consider the estimates (3.6). Due to relation (3.11) and to Theorem 2.2, part 2, we have

$$
S(t, \varepsilon)=o((\varphi * \widetilde{\varphi})(1 / t)), \quad \widetilde{S}(t, \varepsilon)=o((\varphi * \widetilde{\varphi})(1 / t)), \quad t \rightarrow 0+.
$$


Further, the integral in the right-hand side of (3.6) differs from the convolution $(\varphi * \widetilde{\varphi})(\tau)$ by the integrals

$$
\begin{gathered}
\int_{\varepsilon \tau}^{\tau} \varphi\left(\frac{\tau}{\sigma}\right) \widetilde{\varphi}(\sigma) \frac{d \sigma}{\sigma}=\int_{1}^{1 / \varepsilon} \widetilde{\varphi}\left(\frac{\tau}{\sigma}\right) \varphi(\sigma) \frac{d \sigma}{\sigma} \sim \widetilde{\varphi}(\tau) \int_{1}^{1 / \varepsilon} \varphi(\sigma) \frac{d \sigma}{\sigma}=o((\varphi * \widetilde{\varphi})(\tau)), \quad \tau \rightarrow \infty, \\
\int_{1}^{\alpha_{\mp} / \varepsilon} \varphi\left(\frac{\tau}{\sigma}\right) \widetilde{\varphi}(\sigma) \frac{d \sigma}{\sigma} \sim \varphi(\tau) \int_{1}^{\alpha_{\mp} / \varepsilon} \widetilde{\varphi}(\sigma) \frac{d \sigma}{\sigma}=o((\varphi * \widetilde{\varphi})(\tau)), \quad \tau \rightarrow \infty,
\end{gathered}
$$

(we recall that $\tau=\alpha_{ \pm}(\varepsilon) t^{-1}$ ).

Due to Theorem 2.2, part 4, $(\varphi * \widetilde{\varphi})(\tau) \sim(\varphi * \widetilde{\varphi})(1 / t)$, and hence

$$
\Delta_{p, \phi}(\mathcal{T} \otimes \widetilde{\mathcal{T}}) \leq \alpha_{+}(\varepsilon) \frac{a \widetilde{a}}{p}, \quad \delta_{p, \phi}(\mathcal{T} \otimes \widetilde{\mathcal{T}}) \geq \alpha_{-}(\varepsilon) \frac{a \widetilde{a}}{p},
$$

where $\phi$ is defined in (3.13). Taking into account that $\alpha_{ \pm}(\varepsilon) \rightarrow 1$ as $\varepsilon \rightarrow 0$, we arrive at (3.12).

2. Fix arbitrary $\varepsilon>0$ and consider the estimates (3.6). Due to relation (3.14) and Theorem 2.2, part 2, we have

$$
S(t, \varepsilon)=o\left(h_{\widetilde{\varphi}, \varphi}(1 / t)\right), \quad t \rightarrow 0+.
$$

Further, we rewrite the integral in (3.6) as follows:

$$
\int_{\alpha_{\mp}(\varepsilon) / \varepsilon}^{\varepsilon \tau} \varphi\left(\frac{\tau}{\sigma}\right) \widetilde{\varphi}(\sigma) \frac{d \sigma}{\sigma}=h_{\widetilde{\varphi}, \varphi}(\tau)-\int_{1}^{\alpha_{\mp}(\varepsilon) / \varepsilon} \varphi\left(\frac{\tau}{\sigma}\right) \widetilde{\varphi}(\sigma) \frac{d \sigma}{\sigma}+h_{\varphi, \widetilde{\varphi}}(\tau)-\int_{1}^{1 / \varepsilon} \varphi(\sigma) \widetilde{\varphi}\left(\frac{\tau}{\sigma}\right) \frac{d \sigma}{\sigma} .
$$

Due to (3.14) and to Theorem 2.2, part 2, the first integral in the right-hand side is $O(\varphi(\tau))=o\left(h_{\widetilde{\varphi}, \varphi}(\tau)\right)$ as $\tau \rightarrow \infty$, while $\left(2.5_{\varphi \widetilde{\varphi}}\right)$ implies

$$
h_{\varphi, \tilde{\varphi}}(\tau)-\int_{1}^{1 / \varepsilon} \varphi(\sigma) \widetilde{\varphi}\left(\frac{\tau}{\sigma}\right) \frac{d \sigma}{\sigma} \sim \widetilde{\varphi}(\tau) \int_{1 / \varepsilon}^{\infty} \varphi(\sigma) \frac{d \sigma}{\sigma}, \quad \tau \rightarrow \infty .
$$

Finally, (3.7b) and Proposition 2.1, part 5, give us

$$
\widetilde{S}(t, \varepsilon) \sim \widetilde{a} \widetilde{\varphi}(1 / t) \cdot\left(\sum_{n} \lambda_{n}^{1 / p}+\nu(\varepsilon)\right), \quad t \rightarrow 0,
$$

where $\nu(\varepsilon) \rightarrow 0$ as $\varepsilon \rightarrow 0$.

Substituting all these estimates in (3.6) we obtain

$$
\mathcal{N}_{\otimes}(t) \lessgtr \alpha_{ \pm}(\varepsilon) \frac{\phi(\tau)}{t^{1 / p}},
$$

where $\phi$ is defined in (3.15). Due to Theorem 2.2, part $\mathbf{4}, \phi(\tau) \sim \phi(1 / t)$, and hence

$$
\Delta_{p, \phi}(\mathcal{T} \otimes \tilde{\mathcal{T}}) \leq \alpha_{+}(\varepsilon), \quad \delta_{p, \phi}(\mathcal{T} \otimes \widetilde{\mathcal{T}}) \geq \alpha_{-}(\varepsilon) .
$$

Passing to the limit as $\varepsilon \rightarrow 0$ we arrive at (3.12). The proof of part $\mathbf{3}$ is similar.

Example 3. Let

$$
\mathcal{N}(t) \sim a \cdot \frac{\ln ^{\varkappa_{1}}(1 / t)}{t^{1 / p}}, \quad \tilde{\mathcal{N}}(t) \sim \widetilde{a} \cdot \frac{\ln ^{\varkappa_{2}}(1 / t)}{t^{1 / p}}, \quad t \rightarrow 0+.
$$


Then the asymptotic formula (3.12) holds true with

$$
\phi(s)= \begin{cases}\frac{a \tilde{a}}{p} \cdot \mathbf{B}\left(\varkappa_{1}+1, \varkappa_{2}+1\right) \ln ^{\varkappa_{1}+\varkappa_{2}+1}(s), & \varkappa_{1}>-1, \varkappa_{2}>-1 ; \\ \frac{a \widetilde{a}}{p} \cdot \ln (\ln (s)) \cdot \ln ^{\varkappa_{2}}(s), & \varkappa_{1}=-1, \varkappa_{2}>-1 ; \\ \frac{2 a \tilde{a}}{p} \cdot \ln (\ln (s)) \ln ^{-1}(s), & \varkappa_{1}=\varkappa_{2}=-1 ; \\ \widetilde{a} \sum_{n} \lambda_{n}^{1 / p} \cdot \ln ^{\varkappa_{2}}(s), & \varkappa_{1}<-1, \varkappa_{1}<\varkappa_{2} ; \\ \left(a \sum_{n} \widetilde{\lambda}_{n}^{1 / p}+\widetilde{a} \sum_{n} \lambda_{n}^{1 / p}\right) \cdot \ln ^{\varkappa_{1}}(s), & \varkappa_{1}=\varkappa_{2}<-1 .\end{cases}
$$

The particular case $\varkappa_{1}=0, \varkappa_{2}=0$ can be extracted from [PW], see also [LuP2].

Later we use the following inequalities for the functionals $\Delta, \delta$ :

Proposition 3.5. Let $\mathcal{Q}$ and $\mathcal{Q}_{1}$ be compact operators and let $\varphi$ be a SVF. Then

$$
\begin{gathered}
\left|\Delta_{p, \varphi}^{\frac{p}{p+1}}(|\mathcal{Q}|)-\Delta_{p, \varphi}^{\frac{p}{p+1}}\left(\left|\mathcal{Q}_{1}\right|\right)\right| \leq \Delta_{p, \varphi}^{\frac{p}{p+1}}\left(\left|\mathcal{Q}-\mathcal{Q}_{1}\right|\right) ; \\
\left|\delta_{p, \varphi}^{\frac{p}{p+1}}(|\mathcal{Q}|)-\delta_{p, \varphi}^{\frac{p}{p+1}}\left(\left|\mathcal{Q}_{1}\right|\right)\right| \leq \Delta_{p, \varphi}^{\frac{p}{p+1}}\left(\left|\mathcal{Q}-\mathcal{Q}_{1}\right|\right)
\end{gathered}
$$

(we recall that $|\mathcal{Q}|=\sqrt{\mathcal{Q}^{*} \mathcal{Q}}$, see, e.g., $[\mathrm{RS}], \S V I .4$ ).

If $\mathcal{A}$ is a bounded operator, then

$$
\Delta_{p, \varphi}(|\mathcal{A} Q|) \leq\|\mathcal{A}\|^{1 / p} \cdot \Delta_{p, \varphi}(|\mathcal{Q}|) .
$$

Proof. The inequality (3.17a) is a triangle inequality for the functional $\Delta_{p, \varphi}^{\frac{p}{p+1}}$. The inequalities (3.17a), (3.17b) strengthen the well-known Weyl's theorem ([We], see also [BS1], Lemma 1.17). They mean that the functionals $\Delta_{p, \varphi}$ and $\delta_{p, \varphi}$ are continuous in a certain sense. These inequalities are well-known for $\varphi=1$ (see [RSSh], Subsection 11.1; the proofs can be found also in [BS1], Lemma 1.18). The general case was treated in [BS3], Lemma 3.1.

The inequality (3.18) is a simple consequence of variational principle (see, e.g., [BS2], Sec.10.2). As $|\mathcal{A} Q| \leq \| \mathcal{A}|| \cdot|\mathcal{Q}|$, we have

$$
\mathcal{N}(t,|\mathcal{A} Q|) \leq \mathcal{N}(t,|| \mathcal{A}|| \cdot|\mathcal{Q}|) \quad \Longrightarrow \quad \Delta_{p, \varphi}(|\mathcal{A} Q|) \leq \Delta_{p, \varphi}(|| \mathcal{A} \| \cdot|\mathcal{Q}|),
$$

and formula (3.2) yields (3.18).

The next theorem deals with the sum of tensor products of compact operators. Let $\mathcal{T}_{1}, \mathcal{T}_{2}$ and $\widetilde{\mathcal{T}}_{1}, \widetilde{\mathcal{T}}_{2}$ be compact self-adjoint nonnegative operators in Hilbert spaces $H$ and $\widetilde{H}$ respectively. We consider the operators $\mathcal{S}_{1}=\mathcal{T}_{1} \otimes \widetilde{\mathcal{T}}_{1}$ and $\mathcal{S}_{2}=$ $\mathcal{T}_{2} \otimes \widetilde{\mathcal{T}}_{2}$ in the space $H \otimes \widetilde{H}$.

Theorem 3.6. The following statements are true:

1. Let $\varphi$ be a SVF. If, as $t \rightarrow 0+$,

$$
\mathcal{N}\left(t, \mathcal{S}_{1}\right) \sim a t^{-1 / p} \varphi(1 / t), \quad \mathcal{N}\left(t, \mathcal{S}_{2}\right)=o\left(\mathcal{N}\left(t, \mathcal{S}_{1}\right)\right),
$$

then

$$
\mathcal{N}\left(t, \mathcal{S}_{1}+\mathcal{S}_{2}\right) \sim \mathcal{N}\left(t, \mathcal{S}_{1}\right)
$$

2. Let $\varphi_{1}$ and $\widetilde{\varphi}_{2}$ be SVFs. If, as $t \rightarrow 0+$,

$$
\mathcal{N}\left(t, \mathcal{T}_{1}\right) \sim a t^{-1 / p} \varphi_{1}(1 / t), \quad \mathcal{N}\left(t, \widetilde{\mathcal{T}}_{2}\right) \sim \widetilde{a} t^{-1 / p} \widetilde{\varphi}_{2}(1 / t),
$$

and the operators $\widetilde{\mathcal{T}}_{1}, \mathcal{T}_{2}$ are finite-dimensional, then

$$
\mathcal{N}\left(t, \mathcal{S}_{1}+\mathcal{S}_{2}\right) \sim \mathcal{N}\left(t, \mathcal{S}_{1}\right)+\mathcal{N}\left(t, \mathcal{S}_{2}\right) \sim t^{-1 / p} \phi(1 / t), \quad t \rightarrow 0+,
$$


where

$$
\phi(s)=a \varphi_{1}(s) \sum_{n} \lambda_{n}^{1 / p}\left(\widetilde{\mathcal{T}}_{1}\right)+\widetilde{a} \widetilde{\varphi}_{2}(s) \sum_{n} \lambda_{n}^{1 / p}\left(\mathcal{T}_{2}\right) .
$$

3. Let $\varphi$ be a SVF. If, as $t \rightarrow 0+$,

$$
\begin{gathered}
\mathcal{N}\left(t, \mathcal{T}_{1}\right) \sim a t^{-1 / p} \varphi(1 / t), \quad \mathcal{N}\left(t, \widetilde{\mathcal{T}}_{2}\right) \sim \widetilde{a} t^{-1 / p} \varphi(1 / t), \\
\mathcal{N}\left(t, \widetilde{\mathcal{T}}_{1}\right)=O\left(t^{-1 / \widetilde{p}}\right), \quad \mathcal{N}\left(t, \mathcal{T}_{2}\right)=O\left(t^{-1 / \widetilde{p}}\right), \quad \widetilde{p}>p,
\end{gathered}
$$

then

$$
\mathcal{N}\left(t, \mathcal{S}_{1}+\mathcal{S}_{2}\right) \sim \mathcal{N}\left(t, \mathcal{S}_{1}\right)+\mathcal{N}\left(t, \mathcal{S}_{2}\right) \sim A t^{-1 / p} \varphi(1 / t), \quad t \rightarrow 0+,
$$

where

$$
A=a \sum_{n} \lambda_{n}^{1 / p}\left(\widetilde{\mathcal{T}}_{1}\right)+\widetilde{a} \sum_{n} \lambda_{n}^{1 / p}\left(\mathcal{T}_{2}\right)
$$

Proof. 1. This statement is a variant of Weyl's theorem. Actually it is a simple corollary of Proposition 3.5: as $\Delta_{p, \varphi}\left(\mathcal{S}_{2}\right)=0$, we have using (3.17a), (3.17b)

$$
\Delta_{p, \varphi}\left(\mathcal{S}_{1}+\mathcal{S}_{2}\right)=\Delta_{p, \varphi}\left(\mathcal{S}_{1}\right), \quad \delta_{p, \varphi}\left(\mathcal{S}_{1}+\mathcal{S}_{2}\right)=\delta_{p, \varphi}\left(\mathcal{S}_{1}\right)
$$

and the statement follows.

In particular, we see that for any finite-dimensional operator $\mathcal{K}=\mathcal{K}^{*} \geq 0$

$$
\mathcal{N}(t, \mathcal{T}+\mathcal{K}) \sim \mathcal{N}(t, \mathcal{T}), \quad t \rightarrow 0+.
$$

2. Let $P$ be the orthogonal projector in $H$ onto the subspace $\operatorname{ker}\left(\mathcal{T}_{2}\right)$. Correspondingly, denote by $\widetilde{P}$ the orthogonal projector in $\widetilde{H}$ onto $\operatorname{ker}\left(\widetilde{\mathcal{T}}_{1}\right)$. Put

$$
\mathcal{R}_{1}=\left(P \mathcal{T}_{1} P\right) \otimes \widetilde{\mathcal{T}}_{1}, \quad \mathcal{R}_{2}=\mathcal{T}_{2} \otimes\left(\widetilde{P} \widetilde{\mathcal{T}}_{2} \widetilde{P}\right) .
$$

Then $\mathcal{S}_{1}-\mathcal{R}_{1}=\left((I-P) \mathcal{T}_{1}+P \mathcal{T}_{1}(I-P)\right) \otimes \widetilde{\mathcal{T}}_{1}$, and therefore

$$
\operatorname{rank}\left(\mathcal{S}_{1}-\mathcal{R}_{1}\right) \leq 2 \cdot \operatorname{rank}(I-P) \cdot \operatorname{rank} \widetilde{\mathcal{T}}_{1}<\infty .
$$

Similarly, $\operatorname{rank}\left(\mathcal{S}_{2}-\mathcal{R}_{2}\right)<\infty$, and by $(3.21)$

$$
\mathcal{N}\left(t, \mathcal{S}_{i}\right) \sim \mathcal{N}\left(t, \mathcal{R}_{i}\right), \quad t \rightarrow 0+, \quad i=1,2
$$

By construction the operators $\mathcal{R}_{1}$ and $\mathcal{R}_{2}$ are orthogonal, i.e. $\mathcal{R}_{1} \mathcal{R}_{2}=\mathcal{R}_{2} \mathcal{R}_{1}=0$. Hence due to the variational principle we get

$$
\mathcal{N}\left(t, \mathcal{R}_{1}+\mathcal{R}_{2}\right)=\mathcal{N}\left(t, \mathcal{R}_{1}\right)+\mathcal{N}\left(t, \mathcal{R}_{2}\right)
$$

As $\left(\mathcal{S}_{1}+\mathcal{S}_{2}\right)-\left(\mathcal{R}_{1}+\mathcal{R}_{2}\right)$ is also the finite-dimensional operator, we have

$$
\mathcal{N}\left(t, \mathcal{S}_{1}+\mathcal{S}_{2}\right) \sim \mathcal{N}\left(t, \mathcal{R}_{1}+\mathcal{R}_{2}\right) \sim \mathcal{N}\left(t, \mathcal{R}_{1}\right)+\mathcal{N}\left(t, \mathcal{R}_{2}\right) \sim \mathcal{N}\left(t, \mathcal{S}_{1}\right)+\mathcal{N}\left(t, \mathcal{S}_{2}\right),
$$

and (3.19) is proved.

3. Consider the sequences $\mathcal{P}_{j}$ and $\widetilde{\mathcal{P}}_{j}$ of orthogonal projectors onto the first $j$ eigenvectors of $\mathcal{T}_{2}$ and $\widetilde{\mathcal{T}}_{1}$, respectively. Put

$$
\mathcal{Q}_{1 j}=\mathcal{T}_{1} \otimes\left(\widetilde{\mathcal{P}}_{j} \widetilde{\mathcal{T}}_{1} \widetilde{\mathcal{P}}_{j}\right), \quad \mathcal{Q}_{2 j}=\left(\mathcal{P}_{j} \mathcal{T}_{2} \mathcal{P}_{j}\right) \otimes \widetilde{\mathcal{T}}_{2}
$$

According to (3.19) we have

$$
\begin{aligned}
\Delta_{p, \varphi}\left(\mathcal{Q}_{1 j}+\mathcal{Q}_{2 j}\right) & =\Delta_{p, \varphi}\left(\mathcal{Q}_{1 j}\right)+\Delta_{p, \varphi}\left(\mathcal{Q}_{2 j}\right) \\
\delta_{p, \varphi}\left(\mathcal{Q}_{1 j}+\mathcal{Q}_{2 j}\right) & =\delta_{p, \varphi}\left(\mathcal{Q}_{1 j}\right)+\delta_{p, \varphi}\left(\mathcal{Q}_{2 j}\right) .
\end{aligned}
$$

By Theorem 3.2

$$
\Delta_{p, \varphi}\left(\mathcal{S}_{1}-\mathcal{Q}_{1 j}\right)=a \sum_{n} \lambda_{n}^{1 / p}\left(\widetilde{\mathcal{T}}_{1}-\widetilde{\mathcal{P}}_{j} \widetilde{\mathcal{T}}_{1} \widetilde{\mathcal{P}}_{j}\right)=a \sum_{n>j} \lambda_{n}^{1 / p}\left(\widetilde{\mathcal{T}}_{1}\right)^{1 / p} \rightarrow 0, \quad j \rightarrow \infty
$$


Using (3.17a), (3.17b) we have, as $j \rightarrow \infty$,

$$
\Delta_{p, \varphi}\left(\mathcal{Q}_{i j}\right) \rightarrow \Delta_{p, \varphi}\left(\mathcal{S}_{i}\right), \quad \delta_{p, \varphi}\left(\mathcal{Q}_{i j}\right) \rightarrow \delta_{p, \varphi}\left(\mathcal{S}_{i}\right), \quad i=1,2 .
$$

By the triangle inequality (3.17a)

$$
\Delta_{p, \varphi}^{\frac{p}{p+1}}\left(\left(\mathcal{S}_{1}+\mathcal{S}_{2}\right)-\left(\mathcal{Q}_{1 j}+\mathcal{Q}_{2 j}\right)\right) \leq \Delta_{p, \varphi}^{\frac{p}{p+1}}\left(\mathcal{S}_{1}-\mathcal{Q}_{1 j}\right)+\Delta_{p, \varphi}^{\frac{p}{p+1}}\left(\mathcal{S}_{2}-\mathcal{Q}_{2 j}\right),
$$

so that by $(3.17 \mathrm{a}),(3.17 \mathrm{~b})$

$$
\Delta_{p, \varphi}\left(\mathcal{Q}_{1 j}+\mathcal{Q}_{2 j}\right) \rightarrow \Delta_{p, \varphi}\left(\mathcal{S}_{1}+\mathcal{S}_{2}\right), \quad \delta_{p, \varphi}\left(\mathcal{Q}_{1 j}+\mathcal{Q}_{2 j}\right) \rightarrow \delta_{p, \varphi}\left(\mathcal{S}_{1}+\mathcal{S}_{2}\right) .
$$

Passage to the limit in (3.22) completes the proof.

Remark 4. It is easy to see that the condition (3.20) can be weakened. For instance, one can assume that

$$
\mathcal{N}\left(t, \widetilde{\mathcal{T}}_{1}\right)=o\left(t^{-1 / p} \varphi(1 / t)\right), \quad \mathcal{N}\left(t, \mathcal{T}_{2}\right)=o\left(t^{-1 / p} \varphi(1 / t)\right), \quad t \rightarrow 0+,
$$

and both tensor products $\mathcal{S}_{1}$ and $\mathcal{S}_{2}$ satisfy the relations (3.12) and (3.16).

\section{Small Ball asymptotics and the Rate of Eigenvalues DeCREASE}

In this section we connect the given asymptotic behavior of eigenvalues $\lambda_{n}$ from (1.4) with the logarithmic rate of decrease for small deviations. The case $\lambda_{n}=$ $C n^{-p}, p>1$, is now a classical one and is known from [DH-JS], [I], [Z1], [Z2]; see also [DLL], where the exact asymptotics is obtained. The generalization for the case $\lambda_{n}=C n^{-p}(1+o(1)), n \rightarrow \infty$, can be found in [NaNi2], Proposition 2.1, and in $[\mathrm{Fa}]$.

As explained in the introduction, we consider the general case when

$$
\lambda_{n}=C^{*} \frac{\psi(n)}{n^{p}}(1+o(1)), \quad n \rightarrow \infty,
$$

where $p>1$, and the positive function $\psi$ is twice differentiable and satisfies (2.3). We will use the following result which is a concretization of Corollary 3.2 in [DLL] under our conditions.

Lemma 4.1. Denote, for $t, u \geq 0$ and $p>1$

$$
\begin{gathered}
\Phi(t)=t^{-p} \psi(t), \quad f(t)=(1+2 t)^{-1 / 2}, \\
I_{0}(u)=\int_{1}^{\infty} \ln f(u \Phi(t)) d t, \quad I_{1}(u)=u I_{0}^{\prime}(u), \quad I_{2}(u)=u^{2} I_{2}^{\prime \prime}(u) .
\end{gathered}
$$

Then, as $r \rightarrow 0$,

$$
P\left\{\sum_{n=1}^{\infty} \Phi(n) \xi_{n}^{2} \leq r\right\} \sim C_{\Phi} \sqrt{\frac{f(u \Phi(1))}{I_{2}(u)}} \cdot \exp \left(I_{0}(u)+u r\right),
$$

where the constant $C_{\Phi}$ can be expressed in terms of $\Phi$ while $u=u(r)$ is any function satisfying

$$
\lim _{r \rightarrow 0} \frac{I_{1}(u)+u r}{\sqrt{I_{2}(u)}}=0
$$


Note that (2.3) provides logarithmic convexity of $\Phi$ for large values of argument; so all regularity conditions assumed in [DLL] are fulfilled.

All the quantities given above require long but relatively simple calculations. We begin by the asymptotic analysis of $I_{1}(u)$ as $u \rightarrow \infty$ because this integral plays the key role in the sequel. In our case we clearly have

$$
I_{1}(u)=-u \int_{1}^{\infty} \frac{\psi(t) d t}{t^{p}+2 u \psi(t)} .
$$

Changing the integration to $(0, \infty)$ and making a substitution

$$
t=z \Phi^{-1}(1 / u) \equiv z u^{1 / p} \varphi(u)
$$

we get

$$
\begin{gathered}
I_{1}(u)=-u^{1 / p} \varphi(u) \cdot \int_{0}^{\infty} \frac{d z}{2+z^{p} \frac{(\varphi(u))^{p}}{\psi\left(z u^{1 / p} \varphi(u)\right)}}+O(1) \\
=-u^{1 / p} \varphi(u) \cdot \int_{0}^{\infty} \frac{d z}{2+z^{p} \frac{\psi\left(u^{1 / p} \varphi(u)\right)}{\psi\left(z u^{1 / p} \varphi(u)\right)}}+O(1), \quad u \rightarrow \infty .
\end{gathered}
$$

By Proposition 2.1, part 3, for any $\varepsilon>0$ the quotient $\psi(s) / s^{\varepsilon}$ decreases for large $s$. Hence for $z>1, s>1$ we have

$$
\frac{\psi(s)}{\psi(z s)}=\frac{1}{z^{\varepsilon}} \cdot \frac{\psi(s)}{s^{\varepsilon}} / \frac{(z s)^{\varepsilon}}{\psi(z s)} \geq \frac{C(\varepsilon)}{z^{\varepsilon}} .
$$

This estimate gives us the majorant required in the Lebesgue Theorem. Passing to the limit in the integral we obtain as $u \rightarrow \infty$

$$
I_{1}(u) \sim-u^{1 / p} \varphi(u) \cdot \int_{0}^{\infty} \frac{d z}{2+z^{p}}=-u^{1 / p} \varphi(u) \cdot \frac{2^{1 / p} \pi}{2 p \sin \frac{\pi}{p}},
$$

where the last equality follows from [GR], formula 3.241.2. Analogously we get

$$
I_{2}(u)=2 u^{2} \int_{1}^{\infty} \frac{(\psi(t))^{2} d t}{\left(t^{p}+2 u \psi(t)\right)^{2}} \sim-c I_{1}(u), \quad u \rightarrow \infty .
$$

Now note that

$$
\left(\frac{I_{1}(u)}{u}\right)^{\prime}=\frac{I_{2}(u)}{u^{2}}>0, \quad u>0 .
$$

Hence by the implicit function theorem the equation $I_{1}(u)+u r=0$ has for sufficiently small $r$ the unique solution $u(r)$ such that $u(r) \rightarrow \infty$ as $r \rightarrow 0$. Moreover the relation

$$
r=-\frac{I_{1}(u)}{u} \sim \frac{2^{1 / p} \pi}{2 p \sin \frac{\pi}{p}} \cdot u^{-\frac{p-1}{p}} \varphi(u) \equiv \widetilde{\Phi}(u), \quad u \rightarrow \infty,
$$

yields

$$
u(r) \sim \widetilde{\Phi}^{-1}(r) \equiv r^{-\frac{p}{p-1}} \zeta(1 / r), \quad r \rightarrow 0 .
$$


As $u(r)$ trivially satisfies (4.3) we can apply the formula (4.2) with $u=u(r)$. Note that in contrast to the case $\psi \equiv$ const the replacement of $u(r)$ by one-term asymptotics (4.5) breaks the relation (4.3). Therefore it is not fit to extract the exact small ball asymptotics from (4.2). Happily, it fits for the logarithmic asymptotics. Namely, we have

$$
I_{0}(u)=\frac{1}{2} \ln (1+2 u \Phi(1))+u \int_{1}^{\infty} \frac{-p \psi(t)+t \psi^{\prime}(t)}{t^{p}+2 u \psi(t)} d t .
$$

Changing the integration to $(0, \infty)$, making the substitution (4.4) and taking into account the first relation in $(2.3)$, we get

$$
I_{0}(u)=I_{1}(u) \cdot(p+o(1)), \quad u \rightarrow \infty .
$$

Therefore, as $r \rightarrow 0$ and $u=u(r)$,

$$
\ln P\left\{\sum_{n=1}^{\infty} \Phi(n) \xi_{n}^{2} \leq r\right\} \sim I_{0}(u)+u r \sim-(p-1) u r \sim-(p-1) r^{-\frac{1}{p-1}} \zeta(1 / r),
$$

where the SVF $\zeta$ is defined in (4.5).

Now we are able to formulate the following useful statement.

Theorem 4.2. Let the eigenvalues $\lambda_{n}$ from (1.4) have the asymptotics (4.1). Then, as $\varepsilon \rightarrow 0$,

$$
\ln P\left\{\|X\|_{\rho} \leq \varepsilon\right\} \sim-(p-1)\left(C^{*}\right)^{\frac{1}{p-1}} \varepsilon^{-\frac{2}{p-1}} \zeta\left(\varepsilon^{-2}\right),
$$

where the $S V F \zeta$ is defined in (4.5).

For $\psi \equiv$ const this statement is known, see, e.g., [NaNi2] or [Fa]. The proof in the general case is very similar to [NaNi2], Proposition 2.1, and we omit it.

Example 4. Let $\psi(s)=\ln ^{\sigma}(s+1), \sigma \in \mathbb{R}$. The calculation using (4.4) and (4.5) yields

$$
\begin{gathered}
\varphi(u) \sim p^{-\sigma / p} \cdot \ln ^{\sigma / p}(u), \quad u \rightarrow \infty ; \\
\zeta(1 / r) \sim \frac{1}{2}\left(\frac{\pi}{p \sin \frac{\pi}{p}}\right)^{\frac{p}{p-1}}\left(\frac{\ln (1 / r)}{p-1}\right)^{\frac{\sigma}{p-1}}, \quad r \rightarrow 0,
\end{gathered}
$$

and we arrive at

Proposition 4.3. Let the eigenvalues $\lambda_{n}$ from (1.4) have the asymptotics

$$
\lambda_{n} \sim C^{*} \frac{\ln ^{\sigma}(n+1)}{n^{p}}, \quad n \rightarrow \infty, \quad \sigma \in \mathbb{R}, \quad p>1, \quad C^{*}>0 .
$$

Then

$\lim _{\varepsilon \rightarrow 0} \varepsilon^{\frac{2}{p-1}} \ln ^{-\frac{\sigma}{p-1}}(1 / \varepsilon) \cdot \ln P\left\{\|X\|_{\rho} \leq \varepsilon\right\}=-\left(C^{*}\right)^{\frac{1}{p-1}}\left(\frac{p-1}{2}\right)^{1-\frac{\sigma}{p-1}}\left(\frac{\pi}{p \sin \frac{\pi}{p}}\right)^{\frac{p}{p-1}}$.

Let us briefly examine a special case $p=1$. In this case, if SVF $\psi$ satisfies the natural condition (2.4), the statement of Lemma 4.1 also holds true. However, one can check that $r=-I_{1}(u) / u$ is a slowly varying function of $u$. Hence, in contrast with the case $p>1$, its one-term asymptotics does not imply the one-term asymptotics of the inverse function $u(r)$. Therefore we cannot obtain the explicit form of logarithmic asymptotics. For the reason of place we restrict ourselves to one example of weaker asymptotics. 
Proposition 4.4. Let the eigenvalues $\lambda_{n}$ from (1.4) have the asymptotics

$$
\lambda_{n} \sim \frac{C^{*}}{n \ln ^{\sigma}(n+1)}, \quad n \rightarrow \infty, \quad \sigma>1, \quad C^{*}>0 .
$$

Then

$$
\lim _{\varepsilon \rightarrow 0} \varepsilon^{\frac{2}{\sigma-1}} \cdot \ln \left|\ln P\left\{\|X\|_{\rho} \leq \varepsilon\right\}\right|=\left(\frac{C^{*}}{\sigma-1}\right)^{\frac{1}{\sigma-1}} .
$$

Proof. Similarly to Theorem 4.2 we can assume that $\lambda_{n}$ are exactly $\frac{C^{*}}{n \ln ^{\sigma}(n+1)}$. Applying Lemma 4.1, we calculate

$$
r=-\frac{I_{1}(u)}{u}=\int_{1}^{\infty} \frac{C^{*} d t}{t \ln ^{\sigma}(t+1)+2 u C^{*}} .
$$

Define $z=z(u)$ such that

$$
z(\ln (z))^{s}=u, \quad \sigma-1<s<\sigma .
$$

Then, as $u \rightarrow \infty$,

$$
\int_{z}^{\infty} \frac{C^{*} d t}{t \ln ^{\sigma}(t+1)+2 u C^{*}} \sim \int_{z}^{\infty} \frac{C^{*}}{t \ln ^{\sigma}(t)} \cdot \frac{d t}{1+\frac{2 u C^{*}}{t \ln ^{\sigma}(t)}} \sim \int_{z}^{\infty} \frac{C^{*} d t}{t \ln ^{\sigma}(t)}=\frac{C^{*}}{(\sigma-1) \ln ^{\sigma-1}(z)},
$$

while

$$
\int_{1}^{z} \frac{C^{*} d t}{t \ln ^{\sigma}(t+1)+2 u C^{*}} \leq \frac{z}{2 u}=o\left(\frac{1}{\ln ^{\sigma-1}(z)}\right)
$$

Thus

$$
r \sim \frac{C^{*}}{(\sigma-1) \ln ^{\sigma-1}(z)} \sim \frac{C^{*}}{(\sigma-1) \ln ^{\sigma-1}(u)} .
$$

Next, as $u \rightarrow \infty$, we calculate

$$
\begin{aligned}
\ln P\left\{\sum_{n=1}^{\infty} \lambda_{n} \xi_{n}^{2} \leq r\right\} & =I_{0}(u)-I_{1}(u)+O(\ln (u))= \\
& =-u \int_{1}^{\infty} \frac{t}{t+1} \cdot \frac{C^{*} \sigma d t}{\ln (t+1)\left(t \ln ^{\sigma}(t+1)+2 u C^{*}\right)}+O(\ln (u)) .
\end{aligned}
$$

Taking $z=z(u)$ from (4.6), we have

$$
\int_{z}^{\infty} \frac{t}{t+1} \cdot \frac{C^{*} \sigma d t}{\ln (t+1)\left(t \ln ^{\sigma}(t+1)+2 u C^{*}\right)} \sim \int_{z}^{\infty} \frac{C^{*} \sigma}{t \ln ^{\sigma+1}(t)} \cdot \frac{d t}{1+\frac{2 u C^{*}}{t \ln ^{\sigma}(t)}} \sim \frac{C^{*}}{\ln ^{\sigma}(z)},
$$

while

$$
\begin{aligned}
\int_{1}^{z} \frac{t}{t+1} \cdot \frac{C^{*} \sigma d t}{\ln (t+1)\left(t \ln ^{\sigma}(t+1)+2 u C^{*}\right)} & \\
& \leq \int_{1}^{z} \frac{\sigma d t}{2 u \ln (t+1)} \sim \frac{\sigma z}{2 u \ln (z)}=o\left(\frac{1}{\ln ^{\sigma}(z)}\right) .
\end{aligned}
$$


Hence

$$
\ln P\left\{\sum_{n=1}^{\infty} \lambda_{n} \xi_{n}^{2} \leq r\right\} \sim-\frac{C^{*} u}{\ln ^{\sigma}(z)} \sim-\frac{C^{*} u}{\ln ^{\sigma}(u)} .
$$

Due to $(4.7)$, we arrive at

$$
\ln \left|\ln P\left\{\sum_{n=1}^{\infty} \lambda_{n} \xi_{n}^{2} \leq r\right\}\right| \sim \ln (u) \sim\left(\frac{C^{*}}{(\sigma-1) r}\right)^{\frac{1}{\sigma-1}}, \quad r \rightarrow 0 .
$$

Replacing $r$ by $\varepsilon^{2}$ completes the proof.

\section{Fields-Products With EQUAL MARginal EXPONENTS}

In this section we deal with the fields of the form

$$
X(x)=\bigotimes_{j=1}^{d} X_{j}\left(x_{j}\right), \quad x=\left(x_{1}, \ldots, x_{d}\right) \in[0,1]^{d},
$$

where $X_{j}$ are independent zero mean Gaussian processes with equal marginal exponents $p_{j}$ in the asymptotic representation (1.6). Also we consider weight functions of the form

$$
\rho(x)=\prod_{j=1}^{d} \rho_{j}\left(x_{j}\right)
$$

In $\S 8$ we examine a more general class of weights.

We start with the fractional Brownian sheet $\mathbb{W}_{d}^{H}(x)$. Its marginal processes (factors) $X_{j}$ are the fractional Brownian motions $W^{H}(H \in] 0,1[$ is the Hurst parameter), and the corresponding covariance function is

$$
G_{\mathbb{W}_{d}^{H}}(x, y)=\frac{1}{2^{d}} \prod_{j=1}^{d}\left(x_{j}^{2 H}+y_{j}^{2 H}-\left|x_{j}-y_{j}\right|^{2 H}\right) .
$$

Proposition 5.1. Let $0<H<1$ and let $\rho$ be a summable nonnegative function on $[0,1]^{d}$ of the form (5.2). Denote $J_{h}=\int_{[0,1]^{d}} \rho(x)^{1 / h} d x$. Then

$$
\begin{gathered}
\lim _{\varepsilon \rightarrow 0} \varepsilon^{1 / H} \ln ^{-(d-1) \frac{2 H+1}{2 H}}(1 / \varepsilon) \cdot \ln P\left\{\left\|\mathbb{W}_{d}^{H}\right\|_{\rho} \leq \varepsilon\right\} \\
=\frac{-J_{2 H+1} H^{2-d} \pi^{1-d}}{(d-1) !(2 H+1) \sin \left(\frac{\pi}{2 H+1}\right)}\left(\frac{J_{2 H+1}(\Gamma(2 H+1) \sin (\pi H))^{d}}{(d-1) !(\pi H)^{d-1}(2 H+1) \sin \left(\frac{\pi}{2 H+1}\right)}\right)^{\frac{1}{2 H}} .
\end{gathered}
$$

Proof. For $d=1$ the statement coincides with Theorem 3.1 in [NaNi2]. It follows from the proof of this theorem that marginal "one-dimensional" operators corresponding to the marginal covariances have the one-term spectral asymptotics

$$
\lambda_{n}^{(j)} \sim C^{(j)} n^{-(2 H+1)}, \quad j=1, \ldots, d,
$$

where $C^{(j)}=\left(J_{2 H+1}^{(j)} / \pi\right)^{2 H+1} \Gamma(2 H+1) \sin (\pi H)$, while $J_{h}^{(j)}=\int_{0}^{1} \rho_{j}(t)^{1 / h} d t$.

Using Example 3 successively $d-1$ times and then applying Example 2, we obtain that

$$
\lambda_{n} \sim \frac{\prod_{j=1}^{d} C^{(j)}}{(d-1) !^{2 H+1}} \cdot \frac{\ln ^{(d-1)(2 H+1)}(n+1)}{n^{2 H+1}}, \quad n \rightarrow \infty .
$$


Since

$$
\prod_{j=1}^{d} C^{(j)}=J_{2 H+1}^{2 H+1}\left(\frac{\Gamma(2 H+1) \sin (\pi H)}{\pi^{2 H+1}}\right)^{d},
$$

then, using Proposition 4.3 with $p=2 H+1$ and $\sigma=(d-1)(2 H+1)$, we arrive at (5.3).

Remark 5. Formula (5.5) in the case $\rho \equiv 1$ was obtained in [LuP2].

As an example we consider the simplest case of Brownian sheet when $H=1 / 2$. Formula (5.3) gives now

$$
\lim _{\varepsilon \rightarrow 0} \varepsilon^{2} \ln ^{-2(d-1)}(1 / \varepsilon) \cdot \ln P\left\{\left\|\mathbb{W}_{d}\right\|_{\rho} \leq \varepsilon\right\}=-\frac{2^{2 d-5}\left(\int_{[0,1]^{d}} \rho(x)^{1 / 2} d x\right)^{2}}{(d-1) !^{2} \pi^{2 d-2}} .
$$

Formula (5.6) (for $\rho \equiv 1$ ) is equivalent to one obtained in [Cs], see also [Li], Example 3. Note that instead of the norming factor $\ln \left(1 / \varepsilon^{2}\right)$ we use the factor $\ln (1 / \varepsilon)$ that explains the seeming difference in constants (for example, when $d=2$ we obtain the constant $-1 /\left(2 \pi^{2}\right)$ while $(1.2)$ gives $\left.-1 /\left(8 \pi^{2}\right)\right)$.

Remark 6. Note, analogously to Remark 3.1 in [NaNi2], that the replacement of any factor in (5.1) by the fractional Brownian bridge $B^{H}\left(x_{j}\right)=W^{H}\left(x_{j}\right)-x_{j} W^{H}(1)$, by the centered fractional Brownian motion $\bar{W}^{H}\left(x_{j}\right)=W^{H}\left(x_{j}\right)-\int_{0}^{1} W^{H}(s) d s$ or by similar process does not influence on the relation (5.3). For example, the fractional Brownian pillow $\mathbb{B}_{d}^{H}(x)=\underset{j=1}{\otimes} B^{H}\left(x_{j}\right)$ satisfies, as $\varepsilon \rightarrow 0$, the relation

$$
\ln P\left\{\left\|\mathbb{B}_{d}^{H}\right\|_{\rho} \leq \varepsilon\right\} \sim \ln P\left\{\left\|\mathbb{W}_{d}^{H}\right\|_{\rho} \leq \varepsilon\right\} .
$$

The same is true in more general examples. Let us consider the "pinned down" fractional Brownian sheet $\dot{\mathbb{W}}_{d}^{H}(x)$ with covariance

$$
G_{\dot{W}_{d}^{H}}(x, y)=G_{\mathbb{W}_{d}^{H}}(x, y)-G_{\mathbb{W}_{d}^{H}}(x, \mathbf{1}) G_{\mathbb{W}_{d}^{H}}(\mathbf{1}, y), \quad x, y \in[0,1]^{d}
$$

(here $\mathbf{1}=(1,1, \ldots, 1))$. For $H=1 / 2$ this Gaussian field arises in the study of multidimensional goodness-of-fit tests, see [Ni] and [vVW] for similar constructions.

The covariance (5.7) differs from $G_{\mathbb{W}_{d}^{H}}(x, y)$ by the finite-dimensional term. By (3.21) this term does not influence the one-term eigenvalues asymptotics, so we have, as $\varepsilon \rightarrow 0$,

$$
\ln P\left\{\left\|\dot{\mathbb{W}}_{d}^{H}\right\|_{\rho} \leq \varepsilon\right\} \sim \ln P\left\{\left\|\mathbb{W}_{d}^{H}\right\|_{\rho} \leq \varepsilon\right\} .
$$

Now we consider the isotropically integrated fractional Brownian sheet

$$
\left(\mathbb{W}_{d}^{H}\right)_{m}(x)=\stackrel{\otimes}{j=1}_{d}\left(W^{H}\right)_{m}\left(x_{j}\right)
$$

where

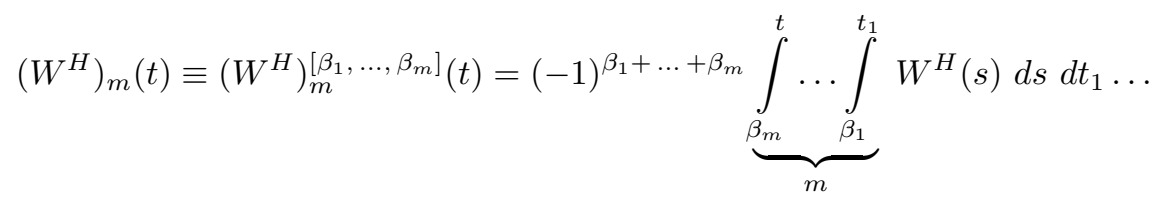

(here any $\beta_{k}$ equals either zero or one, $t \in[0,1]$; for various $j$ the multi-indices $\left[\beta_{1}, \ldots, \beta_{m}\right]$ can differ). See also $[\mathrm{GHT}],[\mathrm{NaNi} 1]$ and $[\mathrm{NaNi} 2]$ for various forms of integrated processes. 
Proposition 5.2. Let $m \in \mathbb{N}$. Then, with the same notation as in Proposition 5.1,

$$
\begin{gathered}
\lim _{\varepsilon \rightarrow 0} \varepsilon^{1 /(m+H)} \ln ^{-(d-1) \frac{2(m+H)+1}{2(m+H)}}(1 / \varepsilon) \cdot \ln P\left\{\left\|\left(\mathbb{W}_{d}^{H}\right)_{m}\right\|_{\rho} \leq \varepsilon\right\} \\
=-\frac{J_{2(m+H)+1}(m+H)^{2-d}}{(d-1) ! \pi^{d-1}(2(m+H)+1) \sin \left(\frac{\pi}{2(m+H)+1}\right)} \\
\cdot\left(\frac{J_{2(m+H)+1}(\Gamma(2 H+1) \sin (\pi H))^{d}}{(d-1) !(\pi(m+H))^{d-1}(2(m+H)+1) \sin \left(\frac{\pi}{2(m+H)+1}\right)}\right)^{\frac{1}{2(m+H)}} .
\end{gathered}
$$

Proof. This statement can be proved in the same way as Proposition 5.1 using Theorem 3.2 in [NaNi2].

The next example deals with the fractional Ornstein - Uhlenbeck sheet $\mathbb{U}_{d,(\alpha)}^{H}(x)$ (here $\alpha \in \mathbb{R}_{+}^{d}$ ). Its marginal processes $X_{j}$ are fractional Ornstein - Uhlenbeck processes $U_{\left(\alpha_{j}\right)}^{H}$. The corresponding covariance function is

$$
G_{\mathbb{U}_{d,(\alpha)}^{H}}(x, y)=\exp \left(-\sum_{j=1}^{d} \alpha_{j}\left|x_{j}-y_{j}\right|^{2 H}\right), \quad 0<H<1 .
$$

In the probabilistic literature there exist different definitions of fractional Ornstein - Uhlenbeck process, here we use the definition from [LuP1]. See the discussion on other possible definitions in [NaNi2], $\S 4$.

The isotropically integrated fractional Ornstein - Uhlenbeck sheet $\left(\mathbb{U}_{d,(\alpha)}^{H}\right)_{m}(x)$ is defined similarly to the isotropically integrated fractional Brownian sheet.

Proposition 5.3. Let $0<H<1, m \in \mathbb{N} \cup\{0\}$ and let $\rho$ be a summable nonnegative function on $[0,1]^{d}$ of the form (5.2). Then, as $\varepsilon \rightarrow 0$,

$$
\ln P\left\{\left\|\left(\mathbb{U}_{d,(\alpha)}^{H}\right)_{m}\right\|_{\rho} \leq \varepsilon\right\} \sim\left(2^{d} \alpha_{1} \ldots \alpha_{d}\right)^{\frac{1}{2(m+H)}} \ln P\left\{\left\|\left(\mathbb{W}_{d}^{H}\right)_{m}\right\|_{\rho} \leq \varepsilon\right\} .
$$

Proof. This statement can be proved in the same way as Proposition 5.1 using Theorem 4.2 from [NaNi2].

We can also consider multiparameter marginal processes. For example, let $\Omega_{j}$, $j=1, \ldots, d$ be bounded domains in $\mathbb{R}^{\ell}$ with $0 \in \overline{\Omega_{j}}$. Set $\Omega=\Omega_{1} \times \Omega_{2} \times \cdots \times \Omega_{d} \subset$ $\mathbb{R}^{d \ell}$. Consider the Gaussian function on $\Omega$

$$
\mathbb{L}_{d, \ell}^{H}(x)=\bigotimes_{j=1}^{d} \mathcal{L}_{\ell}^{H}\left(x_{j}\right),
$$

where $\mathcal{L}_{\ell}^{H}\left(x_{j}\right), 0<H<1$, is Lévy's fractional Brownian motion on $\overline{\Omega_{j}}$ with the covariance

$$
G_{\mathcal{L}_{\ell}^{H}}\left(x_{j}, y_{j}\right)=\frac{1}{2}\left(\left\|x_{j}\right\|^{2 H}+\left\|y_{j}\right\|^{2 H}-\left\|x_{j}-y_{j}\right\|^{2 H}\right), \quad x_{j}, y_{j} \in \overline{\Omega_{j}} .
$$

Proposition 5.4. Let $0<H<1$ and let $\rho$ be a summable nonnegative function on $\Omega$ of the form (5.2). Denote $J_{h}=\int_{\Omega} \rho(x)^{1 / h} d x$. Then

$$
\begin{aligned}
& \lim _{\varepsilon \rightarrow 0} \varepsilon^{\ell / H} \ln ^{-(d-1) \frac{2 H+\ell}{2 H}}(1 / \varepsilon) \cdot \ln P\left\{\left\|\mathbb{L}_{d, \ell}^{H}\right\|_{\rho} \leq \varepsilon\right\} \\
= & -H \cdot\left(\frac{2}{H \Gamma(\ell / 2)}\right)^{d} \cdot\left(\frac{\Gamma(\ell / 2+H)}{\Gamma(\ell / 2) \Gamma(1-H) \pi^{H}}\right)^{\frac{\ell d}{2 H}} \cdot\left(\frac{J_{\frac{2 H+\ell}{\ell}} \pi H(2 H+\ell)^{-1}}{(d-1) ! \sin \left(\frac{\pi \ell}{2 H+\ell}\right)}\right)^{\frac{2 H+\ell}{2 H}} .
\end{aligned}
$$


Proof. This statement can be proved in the same way as Proposition 5.1 using Theorem 3.3 in [NaNi2].

Finally, we can consider the fields-products corresponding to essentially different marginal processes. We restrict ourselves to only one example. Let $H \in] 0,1[$. On $[0,1]^{3}$ consider the Gaussian field $W^{H / 2}\left(x_{1}\right) \otimes \mathcal{L}_{2}^{H}\left(x^{\prime}\right)$, where $x^{\prime}=\left(x_{2}, x_{3}\right) \in[0,1]^{2}$.

Proposition 5.5. With the same notations as in Proposition 5.1,

$$
\begin{gathered}
\lim _{\varepsilon \rightarrow 0} \varepsilon^{2 / H} \ln ^{-\frac{H+1}{H}}(1 / \varepsilon) \cdot \ln P\left\{\left\|W^{H / 2} \otimes \mathcal{L}_{2}^{H}\right\|_{\rho} \leq \varepsilon\right\} \\
=-\frac{J_{H+1}}{\pi(H+1) \sin \left(\frac{\pi}{H+1}\right)}\left(\frac{J_{H+1} H(\Gamma(H+1))^{3} \sin (\pi H / 2) \sin (\pi H)}{4 \pi(H+1) \sin \left(\frac{\pi}{H+1}\right)}\right)^{\frac{1}{H}} .
\end{gathered}
$$

Proof. The spectral asymptotics of marginal operators was established in Theorems 3.1 and 3.3 in [NaNi2]. Applying Example 3, we obtain

$$
\lambda_{n} \sim\left(\frac{J_{H+1}}{\pi^{2}}\right)^{H+1}(\Gamma(1+H))^{3} \sin (\pi H / 2) \sin (\pi H) \cdot \frac{\ln ^{H+1}(n+1)}{n^{H+1}} .
$$

By Proposition 4.3 we immediately arrive at (5.8).

For $H=1 / 2$ and $\rho \equiv 1$ that corresponds to the tensor product of classical nonfractional Lévy's Brownian motion (the so-called isotropic Brownian field) $\mathcal{L}_{2}$ and fractional Brownian motion $W^{1 / 4}$ the result reads

$$
\lim _{\varepsilon \rightarrow 0} \varepsilon^{4} \ln ^{-3}(1 / \varepsilon) \cdot \ln P\left\{\left\|\mathcal{L}_{2} \otimes W^{1 / 4}\right\| \leq \varepsilon\right\}=-\frac{1}{10368 \sqrt{3}} .
$$

\section{FieldS-PROdUCtS With Distinct MARGinal EXPONENTS}

As before we deal with the fields of the form (5.1) but in this section the marginal processes can have different power rates of eigenvalues decrease.

We call $X_{j}$ a slow factor if it has the least exponent $p_{j}$ in (1.5) and call it a fast factor otherwise. Our Theorem 3.2 shows that the rate of eigenvalues decrease for the tensor product is determined only by slow factors. However, in order to calculate the constant in the one-term asymptotics we need rather precise information concerning the fast factors spectra. Using Theorem 4.2 we obtain the following statement.

Lemma 6.1. Let $\lambda_{n}^{(X)}$ and $\lambda_{n}^{(Y)}$ be the eigenvalues of the integral equation (1.4) corresponding to the zero mean Gaussian processes $X$ and $Y$ on $[0,1]$. Suppose the eigenvalues $\lambda_{n}^{(X)}$ satisfy the asymptotics (4.1) while $\lambda_{n}^{(Y)}=O\left(n^{-\widetilde{p}}\right), n \rightarrow \infty$, with $\tilde{p}>p$. Then, as $\varepsilon \rightarrow 0$,

$$
\ln P\{\|\sqrt{\rho} X \otimes Y\| \leq \varepsilon\} \sim\left(\sum_{n}\left(\lambda_{n}^{(Y)}\right)^{1 / p}\right)^{\frac{p}{p-1}} \cdot \ln P\left\{\|X\|_{\rho} \leq \varepsilon\right\} .
$$

Remark 7. Formula (6.1) in a particular case was obtained in [Li], Example 2.

Certainly one can obtain the logarithmic small ball asymptotics for the tensor product with arbitrary number of fast factors applying Lemma 6.1 repeatedly. 
Corollary 6.2. Let the Gaussian random functions $X$ and $Y_{j}, j=1, \ldots, k$, satisfy the conditions of Lemma 6.1. Then, as $\varepsilon \rightarrow 0$,

$$
\ln P\left\{\left\|\sqrt{\rho} X \otimes\left(\otimes_{j=1}^{k} Y_{j}\right)\right\| \leq \varepsilon\right\} \sim \prod_{j=1}^{k}\left(\sum_{n}\left(\lambda_{n}^{\left(Y_{j}\right)}\right)^{1 / p}\right)^{\frac{p}{p-1}} \cdot \ln P\left\{\|X\|_{\rho} \leq \varepsilon\right\} .
$$

Unfortunately, even if we know exact expression for the eigenvalues of the fast factors, the trace-type sums in (6.1) and (6.2) usually cannot be written out explicitly. Consider the following examples. Let $B$ be the standard Brownian bridge. The eigenvalues of Anderson - Darling process $B(t) / \sqrt{t(1-t)}, t \in[0,1]$, see [AnD], are $\lambda_{n}=\left(n^{2}+n\right)^{-1}, n \geq 1$. The processes $t^{\theta} W(t)$ and $t^{\theta} B(t), \theta>-1, t \in[0,1]$, have the eigenvalues which can be expressed in terms of Bessel functions roots, see [Li], Theorem 6, [DM], Theorem 1.3, and [Na1], Theorem 3.3. In these examples the trace-type sums in (6.1) and (6.2) do not admit explicit expressions.

We give below a number of cases when these sums can be expressed in terms of Riemann zeta-function. For any Gaussian process $X(t), t \in[0,1]$, we introduce the centered process $\bar{X}(t)=X(t)-\int_{0}^{1} X(s) d s$ and the so-called on-line centered process $\widehat{X}(t)=X(t)-t^{-1} \int_{0}^{t} X(s) d s$, see $[\mathrm{KlB}]$.

Proposition 6.3. Let the eigenvalues $\lambda_{n}$ of the Gaussian random function $X$ from (1.4) have the asymptotics (4.1). If $p \in] 1,2[$ then, as $\varepsilon \rightarrow 0$,

$$
\ln P\{\|\sqrt{\rho} X \otimes B\| \leq \varepsilon\} \sim \pi^{-\frac{2}{p-1}} \zeta^{\frac{p}{p-1}}(2 / p) \cdot \ln P\left\{\|X\|_{\rho} \leq \varepsilon\right\} ;
$$

$\ln P\{\|\sqrt{\rho} X \otimes \bar{B}\| \leq \varepsilon\} \sim 2^{-\frac{2-p}{p-1}} \pi^{-\frac{2}{p-1}} \zeta^{\frac{p}{p-1}}(2 / p) \cdot \ln P\left\{\|X\|_{\rho} \leq \varepsilon\right\} ;$

$\ln P\{\|\sqrt{\rho} X \otimes W\| \leq \varepsilon\} \sim\left(2^{\frac{2}{p}}-1\right)^{\frac{p}{p-1}} \pi^{-\frac{2}{p-1}} \zeta^{\frac{p}{p-1}}(2 / p) \cdot \ln P\left\{\|X\|_{\rho} \leq \varepsilon\right\} ;$

$$
\begin{aligned}
& \ln P\{\|\sqrt{\rho} X \otimes \bar{W}\| \leq \varepsilon\} \sim \pi^{-\frac{2}{p-1}} \zeta^{\frac{p}{p-1}}(2 / p) \cdot \ln P\left\{\|X\|_{\rho} \leq \varepsilon\right\} ; \\
& \ln P\{\|\sqrt{\rho} X \otimes \widehat{W}\| \leq \varepsilon\} \sim \pi^{-\frac{2}{p-1}} \zeta^{\frac{p}{p-1}}(2 / p) \cdot \ln P\left\{\|X\|_{\rho} \leq \varepsilon\right\} .
\end{aligned}
$$

If $p \in] 1,4[$, then, as $\varepsilon \rightarrow 0$,

$$
\begin{gathered}
\ln P\left\{\left\|\sqrt{\rho} X \otimes(\bar{W})_{1}\right\| \leq \varepsilon\right\} \sim \pi^{-\frac{4}{p-1}} \zeta^{\frac{p}{p-1}}(4 / p) \cdot \ln P\left\{\|X\|_{\rho} \leq \varepsilon\right\} ; \\
\ln P\left\{\left\|\sqrt{\rho} X \otimes \overline{B_{1}}\right\| \leq \varepsilon\right\} \sim \pi^{-\frac{4}{p-1} \zeta^{\frac{p}{p-1}}}(4 / p) \cdot \ln P\left\{\|X\|_{\rho} \leq \varepsilon\right\} .
\end{gathered}
$$

If $m \in \mathbb{N}$ and $p \in] 1,2(m+1)[$, then, as $\varepsilon \rightarrow 0$,

$$
\begin{aligned}
\ln P\left\{\left\|\sqrt{\rho} X \otimes(W)_{m}^{[1,0,1, \ldots]}\right\|\right. & \leq \varepsilon\} \\
& \sim\left(2^{\frac{2(m+1)}{p}}-1\right)^{\frac{p}{p-1}} \pi^{-\frac{2(m+1)}{p-1}} \zeta^{\frac{p}{p-1}}\left(\frac{2(m+1)}{p}\right) \cdot \ln P\left\{\|X\|_{\rho} \leq \varepsilon\right\} .
\end{aligned}
$$

Proof. Due to requirements on $p$, it is easy to see that the second factor in all cases satisfies the condition of Lemma 6.1. Therefore one needs only to derive $\sum_{n}\left(\lambda_{n}^{(Y)}\right)^{1 / p}$.

It is well known that $\lambda_{n}^{(B)}=(\pi n)^{-2}$ and $\lambda_{n}^{(W)}=(\pi(n-1 / 2))^{-2}$. The spectrum of the centered Wiener process $\lambda_{n}^{(\bar{W})}=(\pi n)^{-2}$ can be extracted from [D-MY], see also direct calculations in [BNO]. It was proved in [Wa] that the centered Brownian bridge has double spectrum $\lambda_{2 n-1}^{(\bar{B})}=\lambda_{2 n}^{(\bar{B})}=(2 \pi n)^{-2}$. 
Integrated processes were intensively studied in recent years. It was discovered in [BNO] that $\lambda_{n}^{\left((\bar{W})_{1}\right)}=(\pi n)^{-4}$, while in [HN] there was found that $\lambda_{n}^{\left(\overline{B_{1}}\right)}=(\pi n)^{-4}$. The eigenvalues of so-called Euler integrated Wiener process were derived in $[\mathrm{CH}]$ : $\lambda_{n}^{\left((W)_{m}^{[1,0,1, \ldots]}\right)}=(\pi(n-1 / 2))^{-2(m+1)}$.

As for online centered Wiener process, its covariance $G_{\widehat{W}}(t, s)=s^{2} /(3 t) \wedge t^{2} /(3 s)$ was derived in $[\mathrm{KlB}]$. It is easy to check that $G_{\widehat{W}}$ is the Green function of the boundary value problem

$$
-y^{\prime \prime}+\frac{2 y}{t^{2}}=\mu y ; \quad y(0)=\left(y^{\prime}+y\right)(1)=0 .
$$

With regard to the first boundary condition, we see from [GR], formula 8.491.5, that

$$
y(t)=c \sqrt{t} J_{3 / 2}(\sqrt{\mu} t)=c_{1}\left(\frac{\sin (\sqrt{\mu} t)}{\sqrt{\mu} t}-\cos (\sqrt{\mu} t)\right) .
$$

The second boundary condition in (6.3) gives $\mu_{n}=(\pi n)^{2}$ and hence $\lambda_{n}^{(\widehat{W})}=(\pi n)^{-2}$.

Substituting these eigenvalues into (6.1) we obtain all given formulas.

Let us illustrate the Proposition 6.3 by two particular examples.

1. Consider the Gaussian field $W^{H} \otimes \overline{B_{1}}$. Using the asymptotics obtained in [NaNi2], Theorem 3.1, and applying Proposition 6.3 with $p=2 H+1$, we get, as $\varepsilon \rightarrow 0$,

$$
\begin{aligned}
& \ln P\left\{\left\|\sqrt{\rho} W^{H} \otimes \overline{B_{1}}\right\| \leq \varepsilon\right\} \sim-\pi^{-\frac{2}{H}} \zeta^{\frac{2 H+1}{2 H}}\left(\frac{4}{2 H+1}\right) \\
& \cdot \frac{J_{2 H+1} H}{(2 H+1) \sin \left(\frac{\pi}{2 H+1}\right)}\left(\frac{J_{2 H+1} \Gamma(2 H+1) \sin (\pi H)}{(2 H+1) \sin \left(\frac{\pi}{2 H+1}\right)}\right)^{\frac{1}{2 H}} \varepsilon^{-1 / H} .
\end{aligned}
$$

The particular case of this relation for $H=1 / 2$ and $\rho \equiv 1$, which corresponds to the ordinary Brownian motion, reads

$$
\ln P\left\{\left\|W \otimes \overline{B_{1}}\right\| \leq \varepsilon\right\} \sim-\pi^{-4} \zeta^{2}(2) \ln P\{\|W\| \leq \varepsilon\} \sim-\frac{1}{288 \varepsilon^{2}}, \quad \varepsilon \rightarrow 0
$$

and coincides with what follows from Example 2 in $[\mathrm{Li}]$.

2. The next example deals with the random field $W^{H} \otimes B \otimes(\bar{W})_{1}, 0<H \leq 1 / 2$.

If $H<1 / 2$, then we see from Theorem 3.1 in [NaNi2] that our random field satisfies the conditions of Corollary 6.2. Substituting the eigenvalues of fast factors to $(6.2)$, we get finally, as $\varepsilon \rightarrow 0$,

$$
\begin{aligned}
& \ln P\left\{\left\|\sqrt{\rho} W^{H} \otimes B \otimes(\bar{W})_{1}\right\| \leq \varepsilon\right\} \\
& \sim-\pi^{-\frac{3}{H}} \zeta^{\frac{2 H+1}{2 H}}\left(\frac{4}{2 H+1}\right) \zeta^{\frac{2 H+1}{2 H}}\left(\frac{2}{2 H+1}\right) \\
& \cdot \frac{J_{2 H+1} H}{(2 H+1) \sin \left(\frac{\pi}{2 H+1}\right)}\left(\frac{J_{2 H+1} \Gamma(2 H+1) \sin (\pi H)}{(2 H+1) \sin \left(\frac{\pi}{2 H+1}\right)}\right)^{\frac{1}{2 H}} \varepsilon^{-1 / H} .
\end{aligned}
$$


If $H=1 / 2$, then applying successively Proposition 5.1 (with regard to Remark 6) and Proposition 6.3 we obtain, as $\varepsilon \rightarrow 0$,

$$
\begin{aligned}
\ln P\left\{\left\|\sqrt{\rho} W \otimes B \otimes(\bar{W})_{1}\right\| \leq \varepsilon\right\} & \\
& \sim \pi^{-4} \zeta^{2}(2) \ln P\{\| \sqrt{\rho} W \\
& \otimes B \| \leq \varepsilon\} \\
& \sim-\frac{\left(\int_{0}^{1} \rho(t)^{1 / 2} d t\right)^{2}}{72 \pi^{2}} \ln ^{2}(1 / \varepsilon) \varepsilon^{-2} .
\end{aligned}
$$

Even for $\rho \equiv 1$ when the field considered has the exactly known spectrum

$$
\lambda_{j k \ell}=\pi^{-8}(j-1 / 2)^{-2} k^{-2} \ell^{-4}, \quad j, k, \ell \geq 1,
$$

the result is new and is not covered by [Li].

Now we cite an instance when the eigenvalues $\lambda_{n}^{(Y)}$ cannot be written down explicitly but nevertheless the coefficient on the right-hand side of (6.1) can be derived.

Proposition 6.4. Let the eigenvalues $\lambda_{n}$ from (1.4) have the asymptotics (4.1) with $p=2$. Then, as $\varepsilon \rightarrow 0$,

$$
\ln P\left\{\left\|\sqrt{\rho} X \otimes(W)_{3}^{[0,1,1]}\right\| \leq \varepsilon\right\} \sim \frac{1}{144} \cdot \ln P\left\{\|X\|_{\rho} \leq \varepsilon\right\} .
$$

Proof. Denote by $\mathcal{I}$ the operator of integration from zero:

$$
(\mathcal{I} f)(x)=\int_{0}^{x} f(t) d t
$$

It is shown in [Na1], Theorem 4.1, that the function $G_{(W]_{3}^{[0,1,1]}}(t, s)$ is the kernel of the operator $\left(\mathcal{I}^{*}\right)^{2} \mathcal{I}^{2}\left(\mathcal{I}^{*}\right)^{2} \mathcal{I}^{2}$. The square root of this operator is the integral operator $\left(\mathcal{I}^{*}\right)^{2} \mathcal{I}^{2}$ having the kernel

$$
K(t, s)=\frac{1}{2}(1-s)(1-t)(1-s \vee t)-\frac{1}{6}(1-s \vee t)^{3}
$$

(in fact $K(t, s)=G_{(W)_{1}^{[0]}}(1-t, 1-s)$ but it is inessential). Due to the trace formula for integral operators we have

$$
\sum_{n}\left(\lambda_{n}^{\left((W)_{3}^{[0,1,1]}\right)}\right)^{1 / 2}=\operatorname{Tr}\left(\left(\mathcal{I}^{*}\right)^{2} \mathcal{I}^{2}\right)=\int_{0}^{1} K(t, t) d t=\frac{1}{12},
$$

and the statement follows.

\section{FIELDS-SUMS}

We start with the additive processes of the form (1.7). It is clear that the covariance of the Gaussian field $\mathbb{X}$ reads

$$
G_{\mathbb{X}}(x, y)=\sum_{j=1}^{d} G_{X_{j}}\left(x_{j}, y_{j}\right) .
$$

Assume that $\rho \equiv 1$ and that the processes $X_{j}$ have the spectral asymptotics (1.6).

If $d=2$ we can apply Theorem 3.6, part 2. The operators $\mathcal{T}_{1}$ and $\widetilde{\mathcal{T}}_{2}$ in this case are integral operators with kernels $G_{X_{1}}$ and $G_{X_{2}}$, while $\widetilde{\mathcal{T}}_{1}$ and $\mathcal{T}_{2}$ are integral operators with kernel 1, i.e., orthogonal projectors to one-dimensional space of 
constants. Then Theorem 4.2 gives us the small ball asymptotics for $\mathbb{X}$. In the general case we use Theorem 3.6 successively $d-1$ times.

Now we consider some examples.

Proposition 7.1. Let $\left.H_{j} \in\right] 0,1\left[, m_{j} \in \mathbb{Z}_{+}, j=1, \ldots, d\right.$. Then, as $\varepsilon \rightarrow 0$,

$$
\ln P\left\{\left\|\sum_{j=1}^{d}\left(W^{H_{j}}\right)_{m_{j}}\left(x_{j}\right)\right\| \leq \varepsilon\right\} \sim k^{\frac{2(m+H)+1}{2(m+H)}} \ln P\left\{\left\|\left(W^{H}\right)_{m}\right\| \leq \varepsilon\right\},
$$

where $m=\min _{j} m_{j}, H=\min _{j: m_{j}=m} H_{j}$ while $k=\#\left\{j: m_{j}=m, H_{j}=H\right\}$.

Proposition 7.2. Let $\alpha_{j}>0, j=1, \ldots, d$. Then, as $\varepsilon \rightarrow 0$, with the same notations as in Proposition \%.1,

$$
\begin{aligned}
\ln P\left\{\left\|\sum_{j=1}^{d}\left(U_{\left(\alpha_{j}\right)}^{H_{j}}\right)_{m_{j}}\left(x_{j}\right)\right\|\right. & \leq \varepsilon\} \\
& \sim\left(\sum_{\substack{m_{j}=m \\
H_{j}=H}}\left(2 \alpha_{j}\right)^{\frac{1}{2(m+H)+1}}\right)^{\frac{2(m+H)+1}{2(m+H)}} \ln P\left\{\left\|\left(W^{H}\right)_{m}\right\| \leq \varepsilon\right\} .
\end{aligned}
$$

As in $\S 5$, the processes $X_{j}$ can be multiparameter. We consider one example.

Proposition 7.3. Let $\left.x^{(j)}=\left(x_{1}^{(j)}, \ldots, x_{\ell_{j}}^{(j)}\right), \ell_{j} \in \mathbb{N}, H_{j} \in\right] 0,1\left[, m_{j} \in \mathbb{Z}_{+}, j=\right.$ $1, \ldots, d$. Then, as $\varepsilon \rightarrow 0$,

$$
\ln P\left\{\left\|\sum_{j=1}^{d}\left(\mathbb{W}_{\ell_{j}}^{H_{j}}\right)_{m_{j}}\left(x^{(j)}\right)\right\| \leq \varepsilon\right\} \sim k^{\frac{2(m+H)+1}{2(m+H)}} \ln P\left\{\left\|\left(\mathbb{W}_{\ell}^{H}\right)_{m}\right\| \leq \varepsilon\right\},
$$

where $m=\min _{j} m_{j}, H=\min _{j: m_{j}=m} H_{j}, \ell=\max _{\substack{m_{j}=m \\ H_{j}=H}} \ell_{j}$ while $k=\#\left\{j: m_{j}=m, H_{j}=\right.$ $\left.H, \ell_{j}=\ell\right\}$.

All these results can be generalized to the case when the weight function has the form (5.2). We also give only one example in this context.

Proposition 7.4. Let $\rho_{j}, j=1, \ldots, d$, be summable nonnegative functions on $[0,1]$. Then, as $\varepsilon \rightarrow 0$, with the same notation as in Proposition \%.1,

$$
\begin{aligned}
\ln P\left\{\left\|\sum_{j=1}^{d}\left(W^{H_{j}}\right)_{m_{j}}\left(x_{j}\right)\right\|_{\rho} \leq \varepsilon\right\} & \\
& \sim J_{1}^{\frac{1}{2(m+H)}}\left(\sum_{\substack{m_{j}=m \\
H_{j}=H}} \frac{J_{2(m+H)+1}^{(j)}}{\left(J_{1}^{(j)}\right)^{\frac{1}{2(m+H)+1}}}\right)^{\frac{2(m+H)+1}{2(m+H)}} \cdot \ln P\left\{\left\|W^{H}\right\| \leq \varepsilon\right\}
\end{aligned}
$$

(we recall that $J_{h}^{(j)}=\int_{0}^{1} \rho_{j}(t)^{1 / h} d t$ and $\left.J_{h}=\int_{[0,1]^{d}} \rho(x)^{1 / h} d x\right)$.

Finally we study more general sums with infinite-dimensional operators $\widetilde{\mathcal{T}}_{1}$ and $\mathcal{T}_{2}$. Let $X_{j}$ and $Y_{j}, j=1,2$, be independent Gaussian processes on [0,1]. Consider the following Gaussian field on $[0,1]^{2}$ :

$$
\mathbb{X}=X_{1} \otimes Y_{1}+X_{2} \otimes Y_{2} .
$$


If $X_{1}$ and $Y_{2}$ have the same one-term spectral asymptotics (4.1) while $X_{2}$ and $Y_{1}$ are the fast factors, we can apply Theorem 3.6, part $\mathbf{3}$, and then Theorem 4.2. We restrict ourselves to the following example.

Proposition 7.5. Let $0<H<1 / 2$. Then, as $\varepsilon \rightarrow 0$,

$$
\begin{aligned}
\ln P\left\{\| B^{H}\right. & \left.\otimes W+\bar{B} \otimes W^{H} \| \leq \varepsilon\right\} \\
& \sim\left(2^{\frac{2}{2 H+1}}+2^{-\frac{1-2 H}{2 H+1}}-1\right)^{\frac{2 H+1}{2 H}} \pi^{-\frac{1}{H}} \zeta^{\frac{2 H+1}{2 H}}\left(\frac{2}{2 H+1}\right) \cdot \ln P\left\{\left\|W^{H}\right\| \leq \varepsilon\right\} .
\end{aligned}
$$

\section{Some generalizations}

To consider a more general class of weighted norms we need the following abstract result. Here we do not make distinctions between the function $\Psi$ and the corresponding multiplication operator.

Theorem 8.1. Let $\Omega$ and $\widetilde{\Omega}$ be bounded domains in $\mathbb{R}^{n}$ and $\mathbb{R}^{\widetilde{n}}$, correspondingly. Let two compact self-adjoint nonnegative operators $\mathcal{T}$ and $\widetilde{\mathcal{T}}\left(\right.$ in $L_{2}(\Omega)$ and $L_{2}(\widetilde{\Omega})$, correspondingly) satisfy the following conditions:

1. For any cubes $\omega \subset \Omega, \widetilde{\omega} \subset \widetilde{\Omega}$, as $t \rightarrow 0+$,

$$
\begin{aligned}
& \mathcal{N}\left(t, \chi_{\omega} \mathcal{T} \chi_{\omega}\right) \sim \frac{\varphi(1 / t)}{t^{1 / p}} \cdot \int_{\omega} a(x) d x, \\
& \mathcal{N}\left(t, \chi_{\widetilde{\omega}} \widetilde{\mathcal{T}} \chi_{\widetilde{\omega}}\right) \sim \frac{\widetilde{\varphi}(1 / t)}{t^{1 / p}} \cdot \int_{\widetilde{\omega}} \widetilde{a}(\widetilde{x}) d \widetilde{x},
\end{aligned}
$$

where $\chi_{A}$ stands for the indicator of the set $A, a$ and $\widetilde{a}$ are nonnegative continuous functions in $\Omega$ and $\widetilde{\Omega}$, correspondingly, $p>0$ while $\varphi$ and $\widetilde{\varphi}$ are SVFs satisfying (3.11).

2. For any pairs of nonintersecting open cubes $\omega, \omega_{1} \subset \Omega$ and $\widetilde{\omega}, \widetilde{\omega}_{1} \subset \widetilde{\Omega}$

$$
\Delta_{p, \varphi}\left(\left|\chi_{\omega} \mathcal{T} \chi_{\omega_{1}}\right|\right)=0, \quad \Delta_{p, \widetilde{\varphi}}\left(\left|\chi_{\widetilde{\omega}} \widetilde{\mathcal{T}} \chi_{\widetilde{\omega}_{1}}\right|\right)=0 .
$$

Then for any nonnegative function $\Psi \in \mathcal{C}(\overline{\Omega \times \widetilde{\Omega}})$, as $t \rightarrow 0+$,

$$
\mathcal{N}(t, \Psi(\mathcal{T} \otimes \widetilde{\mathcal{T}}) \Psi) \sim \frac{\phi(1 / t)}{t^{1 / p}} \cdot \int_{\widetilde{\Omega}} \int_{\Omega} \Psi^{2 / p}(x, \widetilde{x}) \frac{a(x) \widetilde{a}(\widetilde{x})}{p} d x d \widetilde{x},
$$

where $\phi(\tau)=(\varphi * \widetilde{\varphi})(\tau)$.

Proof. Step 1. Let $\Psi(x, \widetilde{x})=c \chi_{\omega}(x) \chi_{\widetilde{\omega}}(\widetilde{x})$. Then

$$
\Psi(\mathcal{T} \otimes \widetilde{\mathcal{T}}) \Psi=c^{2}\left(\chi_{\omega} \mathcal{T} \chi_{\omega}\right) \otimes\left(\chi_{\widetilde{\omega}} \widetilde{\mathcal{T}} \chi_{\widetilde{\omega}}\right) .
$$

Applying Theorem 3.4, part 1, and taking into account (3.2), we obtain (8.1).

Step 2. Let $\Psi(x, \widetilde{x}) \equiv \Psi_{N}(x, \widetilde{x})=\sum_{m, \widetilde{m}=1}^{N} c_{m \widetilde{m}} \chi_{\omega_{m}}(x) \chi_{\widetilde{\omega}_{\widetilde{m}}}(\widetilde{x})$, where $\left\{\omega_{m}\right\}_{1}^{N}$ and $\left\{\widetilde{\omega}_{\widetilde{m}}\right\}_{1}^{N}$ are disjoint open cubes in $\Omega$ and $\widetilde{\Omega}$, correspondingly. Then

$$
\Psi(\mathcal{T} \otimes \widetilde{\mathcal{T}}) \Psi=\sum_{j, m, \widetilde{\jmath}, \widetilde{m}=1}^{N} c_{j \widetilde{\jmath}} c_{m \widetilde{m}} \mathcal{S}_{j m} \otimes \widetilde{\mathcal{S}}_{\widetilde{\jmath} \widetilde{m}}
$$

where

$$
\mathcal{S}_{j m}=\chi_{\omega_{j}} \mathcal{T} \chi_{\omega_{m}}, \quad \widetilde{\mathcal{S}}_{\widetilde{\jmath} m}=\chi_{\widetilde{\omega}} \widetilde{\mathcal{T}} \chi_{\widetilde{\omega}}
$$


For $j \neq m$ we have $\Delta_{p, \varphi}\left(\left|\mathcal{S}_{j m}\right|\right)=0$. Similarly to the proof of Theorem 3.3 one can show that for all $\widetilde{\jmath}$ and $\widetilde{m}$

$$
\Delta_{p, \phi}\left(\left|\mathcal{S}_{j m} \otimes \widetilde{\mathcal{S}} \widetilde{\jmath m}\right|\right)=0 .
$$

In the same way, $(8.2)$ holds if $\widetilde{\jmath} \neq \widetilde{m}$. Therefore, by (3.17a), (3.17b) we obtain

$$
\lim _{t \rightarrow 0+} \frac{t^{1 / p}}{\phi(1 / t)} \cdot \mathcal{N}(t, \Psi(\mathcal{T} \otimes \widetilde{\mathcal{T}}) \Psi)=\lim _{t \rightarrow 0+} \frac{t^{1 / p}}{\phi(1 / t)} \cdot \mathcal{N}\left(t, \sum_{m, \widetilde{m}=1}^{N} c_{m \widetilde{m}}^{2} \mathcal{S}_{m m} \otimes \widetilde{\mathcal{S}}_{\widetilde{m} \widetilde{m}}\right)
$$

Next, for $(j, \widetilde{\jmath}) \neq(m, \widetilde{m})$ the operators $\mathcal{S}_{j j} \otimes \widetilde{\mathcal{S}}_{\widetilde{\jmath}}$ and $\mathcal{S}_{m m} \otimes \widetilde{\mathcal{S}}_{\widetilde{m} \widetilde{m}}$ are orthogonal. Hence the variational principle yields

$$
\mathcal{N}\left(t, \sum_{m, \widetilde{m}=1}^{N} c_{m \widetilde{m}}^{2} \mathcal{S}_{m m} \otimes \widetilde{\mathcal{S}}_{\widetilde{m} \widetilde{m}}\right)=\sum_{m, \widetilde{m}=1}^{N} \mathcal{N}\left(t, c_{m \widetilde{m}}^{2} \mathcal{S}_{m m} \otimes \widetilde{\mathcal{S}}_{\widetilde{m} \widetilde{m}}\right)
$$

The asymptotics of summands as $t \rightarrow 0+$ was obtained in Step 1. Substituting (8.4) to (8.3) we get

$$
\mathcal{N}(t, \Psi(\mathcal{T} \otimes \widetilde{\mathcal{T}}) \Psi) \sim \frac{\phi(1 / t)}{t^{1 / p}} \cdot \sum_{m, \widetilde{m}=1}^{N} c_{m \widetilde{m}}^{2 / p} \int_{\widetilde{\omega}_{\widetilde{m}}} \int_{\omega_{m}} \frac{a(x) \widetilde{a}(\widetilde{x})}{p} d x d \widetilde{x},
$$

that gives (8.1).

Step 3. Let the function $\Psi$ be uniformly approximated by functions $\Psi_{N}$ considered in Step 2. In view of $(3.17 \mathrm{a}),(3.17 \mathrm{~b})$ for passing to the limit it is sufficient to prove that

$$
\Delta_{p, \phi}\left(\left|\Psi(\mathcal{T} \otimes \widetilde{\mathcal{T}}) \Psi-\Psi_{N}(\mathcal{T} \otimes \widetilde{\mathcal{T}}) \Psi_{N}\right|\right) \rightarrow 0, \quad N \rightarrow \infty .
$$

By (3.17a) and (3.18) we obtain

$$
\begin{gathered}
\left(\Delta_{p, \phi}\left(\left|\Psi(\mathcal{T} \otimes \widetilde{\mathcal{T}}) \Psi-\Psi_{N}(\mathcal{T} \otimes \widetilde{\mathcal{T}}) \Psi_{N}\right|\right)\right)^{\frac{p}{p+1}} \\
\leq\left(\Delta_{p, \phi}\left(\left|\left(\Psi-\Psi_{N}\right)(\mathcal{T} \otimes \widetilde{\mathcal{T}}) \Psi\right|\right)\right)^{\frac{p}{p+1}}+\left(\Delta_{p, \phi}\left(\left|\Psi_{N}(\mathcal{T} \otimes \widetilde{\mathcal{T}})\left(\Psi-\Psi_{N}\right)\right|\right)\right)^{\frac{p}{p+1}} \\
\leq\left\|\Psi-\Psi_{N}\right\|^{\frac{1}{p+1}} \cdot\left(\Delta_{p, \phi}(\mathcal{T} \otimes \widetilde{\mathcal{T}})\right)^{\frac{p}{p+1}} \cdot\left(\|\Psi\|^{\frac{1}{p+1}}+\left\|\Psi_{N}\right\|^{\frac{1}{p+1}}\right) .
\end{gathered}
$$

Here $\|\cdot\|$ stands for the norms of multiplication operators in $L_{2}(\Omega \times \widetilde{\Omega})$ which coincide with $L_{\infty}$-norms of corresponding functions. So, the last product tends to zero as $N \rightarrow \infty$, and (8.1) follows.

It remains to note that the class of weights considered contains $\mathcal{C}(\overline{\Omega \times \widetilde{\Omega}})$.

Corollary 8.2. Propositions 5.1 - 5.4 hold true for any $\rho \in \mathcal{C}\left([0,1]^{d}\right)$.

Proof. First of all, applying Theorem 8.1 repeatedly we obtain analogous result for the tensor product $\underset{j=1}{\otimes} \mathcal{T}_{j}$. Next, due to [BS1], Appendix 7, the marginal integral operators under consideration satisfy the conditions of Theorem 8.1. Then we can put $\Psi=\sqrt{\rho}$, and the statement follows.

Remark 8. In fact, Theorem 8.1 is true for $\Psi \in L_{2}(\Omega \times \widetilde{\Omega})$ (and therefore Corollary 8.2 is true for $\left.\rho \in L_{1}\left([0,1]^{d}\right)\right)$. The proof requires more delicate interpolation techniques. We are going to return to this problem in a separate work.

The results of previous sections can be also generalized to the vector-valued zero mean Gaussian fields $X(x) \in \mathbb{R}^{\ell}, x \in[0,1]^{d}$. Let, for example, the components 
$X^{(\mu)}, \mu=1, \ldots, \ell$, be $d$-dimensional fractional Brownian sheets with the Hurst parameters $H_{\mu}$. Let $A(x)$ be real $\ell \times k$-matrix, $A^{(\mu \tau)}(x)=\prod_{j=1}^{d} A_{j}^{(\mu \tau)}\left(x_{j}\right)$ for all $\mu=1, \ldots, \ell, \tau=1, \ldots, k$, and $A_{j} \in L_{2}([0,1])$. Then

$$
A^{T}(x) X(x)=\stackrel{\otimes}{j=1}_{j} A_{j}^{T} X_{j},
$$

where the marginal processes $X_{j}$ are the vector-valued fractional Brownian motions on $[0,1]$.

Nazarov and Nikitin $([\mathrm{NaNi} 2], \S 5)$ derived the spectral asymptotics for $A_{j}$ weighted vector-valued fractional Brownian motions. It was proved that

$$
\lambda_{n}^{(j)}=\widehat{C}^{(j)} n^{-(2 H+1)}(1+o(1)),
$$

where $H=\min _{\mu} H_{\mu}$ while $\widehat{C}^{(j)}$ can be determined in terms of $A_{j}$ and marginal crosscovariance matrices $G_{j}^{(\mu \nu)}\left(x_{j}, y_{j}\right)=E X_{j}^{(\mu)}\left(x_{j}\right) X_{j}^{(\nu)}\left(y_{j}\right)$. The same arguments as in Proposition 5.1 now yield

$$
\begin{aligned}
\lim _{\varepsilon \rightarrow 0} \varepsilon^{1 / H} \ln ^{-(d-1) \frac{2 H+1}{2 H}}(1 / \varepsilon) \cdot \ln P\left\{\int_{[0,1]^{d}}\left\|A^{T}(x) X(x)\right\|^{2} d x \leq \varepsilon^{2}\right\} \\
=-\left(\frac{\prod_{j=1}^{d} \widehat{C}^{(j)}}{(d-1) !^{2 H+1}}\right)^{\frac{1}{2 H}} H^{1-\frac{(d-1)(2 H+1)}{2 H}}\left(\frac{\pi}{(2 H+1) \sin \left(\frac{\pi}{2 H+1}\right)}\right)^{\frac{2 H+1}{2 H}} .
\end{aligned}
$$

In the case when only one (for example, the first) component $X^{(\mu)}$ has the least Hurst parameter, i.e. $H_{1}=H, H_{\mu}>H$ for $\mu \geq 2$, the explicit formula for $\widehat{C}^{(j)}$ gives, similarly to $(5.6)$,

$$
\prod_{j=1}^{d} \widehat{C}^{(j)}=\left(\mathcal{J}_{2 H+1}^{(1)}\right)^{2 H+1}\left(\frac{\Gamma(2 H+1) \sin (\pi H)}{\pi^{2 H+1}}\right)^{d}
$$

(here $\mathcal{J}_{h}^{(\mu)}=\int_{[0,1]^{d}}\left|A^{(\mu)}(x)\right|^{2 / h} d x$ while $A^{(\mu)}$ is the $\mu$-th row of the matrix $A$ ).

The formula obtained holds true for $A \in \mathcal{C}\left([0,1]^{d} \rightarrow \mathbb{R}^{\ell k}\right)$. It can be proved by applying the vector-valued analogue of Theorem 8.1.

Analogous statements are true for the vector-valued isotropically integrated fractional Brownian sheet, for the vector-valued fractional Ornstein - Uhlenbeck sheet, and for similar fields.

\section{ACKNOWLEDGEMENTS}

We are grateful to Professors M.A. Lifshits and M.Z. Solomyak for valuable consultations.

\section{REFERENCES}

[Ad] R. Adler, An Introduction to Continuity, Extrema and Related Topics for General Gaussian Processes, IMS Lect. Notes - Monograph Series 12, IMS, Hayward, California, 1990. MR1088478 (92g:60053)

[AnD] T.W. Anderson and D.A. Darling, Asymptotic theory of certain "goodness-of-fit" criteria based on stochastic processes, Ann. Math. Statist., 23 (1952), 193-212. MR0050238 $(14: 298 \mathrm{~h})$

[BNO] L. Beghin, Ya.Yu. Nikitin, E. Orsingher, Exact small ball constants for some Gaussian processes under the $L_{2}$-norm, Zap. Nauchn. Sem. St.-Petersburg. Otdel. Mat. Inst. Steklov (POMI), 298 (2003), 5-21. 
[BL] E. Belinsky and W. Linde, Small ball probabilities of fractional Brownian sheets via fractional integration operators, J. Theor. Probab., 15 (2002), 589-612. MR1922439 (2004d:60092)

[BS1] M.S. Birman and M.Z. Solomyak, Quantitative analysis in Sobolev imbedding theorems and applications to spectral theory, In: Proceed. of X Summer Mathematical School, Yu.A. Mitropol'skiy and A.F. Shestopal (Eds), 1974, 5-189 (in Russian). English transl. in: AMS Transl., Ser. 2, 114, AMS, Providence, R.I., 1980. MR562305 (80m:46026)

[BS2] M.S. Birman and M.Z. Solomyak, Spectral Theory of Self-adjoint Operators in Hilbert Space. Leningrad University Publishers, 1980 (in Russian). English transl. in: Math. and Its Applic. Soviet Series, 5, Dordrecht, etc.: Kluwer Academic Publishers, 1987.

[BS3] M.Sh. Birman and M. Solomyak, On the negative discrete spectrum of a periodic elliptic operator in a waveguide-type domain, perturbed by a decaying potential, Journ. d'Analyse Math., 83 (2001), 337-391. MR1828497 (2002k:35226)

[Br] J.C. Bronski, Small ball constants and tight eigenvalue asymptotics for fractional Brownian motions, J. Theor. Probab., 16 (2003), 87-100. MR1956822 (2004b:60105)

[Ca] R. Carmona, Tensor products of Gaussian measures, Lect. Notes in Math., 644 (1977), 96-124. MR502402 (80a:60039)

[CC] R. Carmona and S. Chevet, Tensor Gaussian measures on $L_{p}(E)$, J. Funct. Anal., 33 (1970), 297-310. MR549116 (80j:60010)

[CH] C.-H. Chang and C.-W. Ha, The Green's functions of some boundary value problems via the Bernoulli and Euler polynomials, Arch. Mat., 76 (2001), 360-365. MR1824255 (2002b:34046)

[CL] X. Chen and W.V. Li, Small deviation estimates for some additive processes, Proc. Conf. High Dimensional Probab. III, Progress in Probability, 55 (2003), Birkhäuser, 225-238. MR2033891 (2004m:60115)

[Ch] S. Chevet, Un résultat sur les mesures gaussiennes, C.R. Acad. Sci. Paris Sér. A-B, 284 (1977), A441-A444. MR0428413 (55:1434)

[Cs] E. Csáki, On small values of the square integral of a multiparameter Wiener process,. In: Statistics and Probability, Proc. of the 3rd Pannonian Symp. on Math. Stat. D.Reidel, Boston, 1982, 19-26. MR758997

[DM] P. Deheuvels and G. Martynov, Karhunen - Loève expansions for weighted Wiener processes and Brownian bridges via Bessel functions, Proc. Conf. High Dimensional Probab. III, Progress in Probability, 55 (2003), Birkhäuser, 57-93. MR2033881 (2005i:62074)

[De] S. Dereich, Small ball probabilities around random centers of Gaussian measures with applications to quantization, J. Theor. Probab., 16 (2003), 427-449. MR1982037 (2004d:28033)

[D-MY] C. Donati-Martin and M. Yor, Fubini's theorem for double Wiener integrals and the variance of the Brownian path, Ann. Inst. H. Poincaré, Probab. Stat., 27 (1991), 181200. MR1118933 (92m:60072)

[DH-JS] R.M. Dudley, J. Hoffman-Jørgensen, L.A. Shepp, On the lower tail of Gaussian seminorms, Ann. Prob., 7 (1979), 319-342. MR525057 (80j:60051)

[DLL] T. Dunker, M.A. Lifshits, W. Linde, Small deviations of sums of independent variables, In: Proc. Conf. High Dimensional Probab., Ser. Progress in Probability, Birkhäuser, 43 (1998), 59-74. MR1652320 (2000h:60035)

[Fa] V.R. Fatalov, Constants in the asymptotics of small deviation probabilities for Gaussian processes and fields, Russ. Math. Surv. 58 (2003), 725-772. MR2042263 (2005a:60052)

[FT] J.A. Fill and F. Torcaso, Asymptotic analysis via Mellin transforms for small deviations in $L_{2}$-norm of integrated Brownian sheets, Probab. Theory and Rel. Fields, 130 (2004), 259-288. MR2093764 (2005i:60066)

[GHT] F. Gao, J. Hannig, F. Torcaso, Integrated Brownian motions and exact $L_{2}$-small balls, Ann. Prob., 31 (2003), 1320-1337. MR1989435 (2004k:60104)

[GHLT1] F. Gao, J. Hannig, T.-Y. Lee, F. Torcaso, Laplace transforms via Hadamard factorization with applications to small ball probabilities, Electronic J. Prob., 8 (2003), 1-20. MR1998764 (2005h:60110)

[GHLT2] F. Gao, J. Hannig, T.-Y. Lee, F. Torcaso, Exact $L^{2}$-small balls of Gaussian processes, J. of Theor. Prob., 17 (2004), 503-520. MR2053714 (2005d:60059) 
[GL1] F. Gao and W.V. Li, Small ball probabilities for the Slepian Gaussian fields, Trans. Amer. Math. Soc. 359 (2007), 1339-1350. MR2262853

[GL2] F. Gao and W.V. Li, Logarithmic level comparison for small deviation probabilities, J. Theoret. Probab. 20 (2007), 1-23. MR2297848

[GR] I.S. Gradshteyn and I.M. Ryzhik, Tables of integrals, sums, series and products, 5th ed. Moscow, Nauka, 1971 (in Russian). English transl.: Table of integrals, series, and products. Corr. and enl. ed. by Alan Jeffrey. New York - London - Toronto: Academic Press, 1980. MR582453 (81g:33001)

[GHP] S. Graf, H. Luschgy, H. Pagès, Functional quantization and small ball probabilities for Gaussian processes, J. Theor. Probab., 16 (2003), 1047-1062. MR2033197 (2004k:60105)

[HN] N. Henze and Ya.Yu. Nikitin, Watson-type goodness-of-fit tests based on the integrated empirical process, Mathem. Meth. of Statist., 11 (2002), 183-202. MR1941315 (2003i:62082)

[I] I.A. Ibragimov, The probability of a Gaussian vector with values in a Hilbert space hitting a ball of small radius, Zap. Nauchn. Semin. Leningrad. Otdel. Mat. Inst. Steklov (LOMI), 85 (1979), 75-93. English transl. in: J. Sov. Math. 20 (1982), 2164-2174. MR535459 (81g:60006)

[KNN] A.I. Karol', A.I. Nazarov, Ya.Yu. Nikitin, Tensor products of compact operators and logarithmic $L_{2}$-small ball asymptotics for Gaussian random fields, Studi Statistici No.74, Istituto di Metodi Quantitativi, Università L.Bocconi, Milano, July 2003, 30 pp.

[Kh] D. Khoshnevisan, Multiparameter Processes. An Introduction to Random Fields. Springer Monographs in Mathematics. NY: Springer, 2002. MR1914748 (2004a:60003)

[KlB] M.L. Kleptsyna and A. Le Breton, A Cameron - Martin type formula for general Gaussian processes - a filtering approach, Stochastics and Stoch. Rep., 72 (2002), 229-250. MR1897916 (2003g:60063)

[KLn] T. Kühn and W. Linde, Optimal series representation of fractional Brownian sheets, Bernoulli, 8 (2002), 669-696. MR1935652 (2003m:60131)

[Li] W.V. Li, Comparison results for the lower tail of Gaussian seminorms, J. of Theor. Prob., 5 (1992), 1-31. MR1144725 (93k:60088)

[LiS] W.V. Li and Q.M. Shao, Gaussian processes: inequalities, small ball probabilities and applications, In: Stochastic Processes: Theory and Methods, Handbook of Statistics, 19 (2001), C.R.Rao and D.Shanbhag (Eds), 533-597. MR1861734

[Lf] M.A. Lifshits, Asymptotic behavior of small ball probabilities, In: Prob. Theory and Math. Stat., B.Grigelionis et al. (Eds), Proc. VII International Vilnius Conference, VSP/TEV, 1999, 453-468.

[LNN] M.A. Lifshits, A.I. Nazarov, Ya.Yu. Nikitin, Tail behavior of anisotropic norms for Gaussian random fields, C.R. Acad. Sci. Paris I, 336 (2003), 85-88. MR1968908 (2004b:60132)

[LuP1] H. Luschgy and G. Pagès, Functional quantization of Gaussian processes, J. Funct. Analysis, 196 (2002), 486-531. MR1943099 (2003i:60006)

[LuP2] H. Luschgy and G. Pagès, Sharp asymptotics of the functional quantization problem for Gaussian processes, Ann. Probab., 32 (2004), 1574-1599. MR2060310 (2005d:60036)

[MSh] D.M. Mason and Z. Shi, Small deviations for some multi-parameter Gaussian processes, J. Theor. Probab., 14 (2001), 213-239. MR1822902 (2001m:60091)

[Na1] A.I. Nazarov, On the sharp constant in the small ball asymptotics of some Gaussian processes under $L_{2}$-norm, Problems of Math. Anal., 26 (2003), 179-214 (in Russian). English transl. in: J. of Math. Sci., 117 (2003), 4185-4210. MR2027455 (2004j:60080)

[Na2] A.I. Nazarov, Logarithmic $L_{2}$-small ball asymptotics with respect to self-similar measure for some Gaussian processes, Zap. Nauchn. Semin. St.-Petersb. Otdel. Mat. Inst. Steklov (POMI), 311 (2004), 190-213 (in Russian). To be translated in J. of Math. Sci. MR2092208 (2005j:60078)

[NaNi1] A.I. Nazarov and Ya.Yu. Nikitin, Exact $L_{2}$-small ball behavior of integrated Gaussian processes and spectral asymptotics of boundary value problems, Probab. Theory and Rel. Fields, 129 (2004), 469-494. MR2078979 (2005d:60060)

[NaNi2] A.I. Nazarov and Ya.Yu. Nikitin, Logarithmic $L_{2}$-small ball asymptotics for some fractional Gaussian processes, Theor. Probab. and Appl., 49 (2004), 695-711. MR2142562 (2006b:60070) 
[Ni] Ya. Nikitin, Asymptotic Efficiency of Nonparametric Tests, Cambridge University Press, 1995. MR1335235 (96c:62093)

$[\mathrm{PW}] \quad$ A. Papageorgiou and G.W. Wasilkowski, On the average complexity of multivariate problems, J. of Complexity, 6 (1990), 1-23. MR1048027 (91b:94020)

[RS] M. Reed and B. Simon, Methods of Modern Mathematical Physics. V.1: Functional Analysis, New York - London: Academic Press, Inc. XVII, 1972.

[RWW] K. Ritter, G.W. Wasilkowski, H. Woźniakovski, Multivariate integration and approximation for random fields satisfying Sacks - Ylvisaker conditions, Ann. Appl. Probab., 5 (1995), 518-540. MR1336881 (96d:60073)

[RSSh] G.V. Rosenblum, M.Z. Solomyak, M.A. Shubin, Spectral Theory of Differential Operators. Modern Problems of Mathematics, 64 (1989), Moscow, VINITI, 1-242 (in Russian). Engl. transl. in: Partial Differ. Equations VII. Encycl. Math. Sci. 64 (1994), 1-261. MR1313735 (95j:35156)

[Se] E. Seneta, Regularly Varying Functions. Lect. Notes in Mathem., 508 (1976). MR0453936 (56:12189)

[Sy] G.N. Sytaya, On some asymptotic representations of the Gaussian measure in a Hilbert space, In: Theory of Stochastic Processes, Kiev, 2 (1974), 93-104 (in Russian).

[vVW] A. van der Vaart and J.A. Wellner, Weak Convergence and Empirical Processes with Applications to Statistics, Springer Series in Statistics. NY, Springer, 1986. MR1385671 (97g:60035)

[Wa] G.S. Watson, Goodness-of-fit tests on a circle, Biometrika, 48 (1961), 109-114. MR0131930 (24:A1777)

[We] H. Weyl, Das asymptotische Verteilungsgesetz der Eigenwerte linearer partieller Differentialgleichungen, Math. Ann., 71 (1912), 441-479. MR1511670

[Z1] V.M. Zolotarev, Gaussian measure asymptotics in $l_{2}$ on a set of centered spheres with radii tending to zero, In: 12th Europ. Meeting of Statisticians, Varna, 1979, 254.

[Z2] V.M. Zolotarev, Asymptotic behavior of Gaussian measure in $l_{2}$, Problems of stability of stochastic models, Proc. Semin., Moscow, 1984, 54-58 (in Russian). English transl. in: J. Sov. Math., 24 (1986), 2330-2334. MR859211

Department of Mathematics and Mechanics, St. Petersburg State University, Universitetski Pr., 28, St. Petersburg, 198504, Russia

E-mail address: karol@ak1078.spb.edu

Department of Mathematics and Mechanics, St. Petersburg State University, Universitetski Pr., 28, St. Petersburg, 198504, Russia

E-mail address: an@AN4751.spb.edu

Department of Mathematics and Mechanics, St. Petersburg State University, Universitetski Pr., 28, St. Petersburg, 198504, Russia

E-mail address: yanikit47@mail.ru 